The Canadian Mineralogist

Vol. 42, pp. 1221-1240 (2004)

\title{
THE COMPOSITION OF CO-Ni-Fe SULFARSENIDES, DIARSENIDES AND TRIARSENIDES FROM THE SAN JUAN DE PLAN DEPOSIT, CENTRAL PYRENEES, SPAIN
}

\author{
ISABEL FANLO ${ }^{\S}$ AND IGNACIO SUBÍAS \\ Cristalografía y Mineralogía. Departamento de Ciencias de la Tierra. \\ Universidad de Zaragoza, C/ Pedro Cerbuna 12, E-50009 Zaragoza, Spain \\ FERNANDO GERVILLA \\ Instituto Andaluz de Ciencias de la Tierra y Departamento de Mineralogía y Petrología. \\ Facultad de Ciencias, C.S.I.C.-Universidad de Granada, E-18002 Granada, Spain \\ ANDRÉs PANIAGUA ANd BELÉN GARCÍA \\ Cristalografía y Mineralogía. Departamento de Ciencias de la Tierra. \\ Universidad de Zaragoza, C/ Pedro Cerbuna 12, E-50009 Zaragoza, Spain
}

\begin{abstract}
The San Juan de Plan deposit, in the central Pyrenees of Spain, is situated in an ankeritic horizon in Silurian black shales. The main association consists of pyrite, sulfarsenides, diarsenides, and Co triarsenides (skutterudite), as well as a late stage consisting of cobaltite, $\mathrm{Fe}, \mathrm{Cu}$ and $\mathrm{Bi}$ sulfides and native $\mathrm{Bi}$. The sulfarsenides are arsenopyrite, alloclasite, members of the gersdorffitecobaltite solid-solution series, arsenic-rich gersdorffite and cobaltite, whereas the diarsenides range from rammelsbergite to safflorite. Rammelsbergite is characterized by a composition within the range $\left(\mathrm{Ni}_{0.71-0.97} \mathrm{Co}_{0.02-0.29} \mathrm{Fe}_{0.00-0.02}\right) \mathrm{As}_{1.75-2.01} \mathrm{~S}_{0.00-0.26}$, exceeding the theoretical value $\left(\mathrm{As}_{1.93} \mathrm{~S}_{0.07}\right)$ given by Yund (1962). Two generations of the gersdorffite-cobaltite solid-solution series have been found on the basis of textural features and chemical composition; the first, $\left(\mathrm{Co}_{0.10-0.77} \mathrm{Ni}_{0.07-0.80} \mathrm{Fe}_{0.03-0.58}\right) \mathrm{As}_{0.95-}$ ${ }_{1.27} \mathrm{~S}_{0.75-1.06}$, shows a positive correlation between $\mathrm{S}$ and Fe contents, and between Co and As contents, whereas the second one, $\left(\mathrm{Co}_{0.02-0.91} \mathrm{Ni}_{0.02-0.96} \mathrm{Fe}_{0.00-0.28}\right) \mathrm{As}_{0.99-1.38} \mathrm{~S}_{0.67-1.01}$, exhibits a considerable compositional field in the Fe-poor region of the system CoAsS-NiAsS-FeAsS , providing evidence of extensive substitution of Co by $\mathrm{Ni}$, with a positive correlation between $\mathrm{As}$ and $\mathrm{Ni}$ contents. Arsenic-rich gersdorffite displays a broad range of $\mathrm{As}$ and $\mathrm{S}$ mutual substitution, $\left(\mathrm{Ni}_{0.65-0.98} \mathrm{Co}_{0.02-0.27} \mathrm{Fe}_{0.00-0.06}\right) \mathrm{As}_{1.37-}$ ${ }_{1.83} \mathrm{~S}_{0.22-0.64}$, exceeding the experimental value of $\mathrm{NiAs}_{1.77} \mathrm{~S}_{0.23}$ reported by Yund (1962). The presence of alloclasite and the extent of the solid-solution fields of the cobaltite-gersdorffite series suggest that the main assemblage of minerals at the San Juan de Plan deposit formed at temperatures as high as $600^{\circ} \mathrm{C}$. Preliminary geothermometric data obtained on graphite support such a high temperature.
\end{abstract}

Keywords: Ni-Co-Fe sulfarsenides, gersdorffite-cobaltite solid-solution series, alloclasite, As-rich gersdorffite, rammelsbergite, skutterudite, compositional trends, San Juan de Plan, Spain.

\section{SOMMAIRE}

Le gisement de San Juan de Plan, situé dans les Pyrénées centrales de l'Espagne, se trouve dans un couche ankéritique d'une séquence de shales noirs d'âge silurien. Le minerai est fait surtout de pyrite, de sulfarséniures, de diarséniures et de triarséniures de cobalt (skutterudite), de même que de cobaltite, de sulfures de $\mathrm{Fe}, \mathrm{Cu}$ et $\mathrm{Bi}$, et de bismuth natif, déposés tardivement. Parmi les sulfarséniures se trouvent arsénopyrite, alloclasite, des membres de la solution solide gersdorffite-cobaltite, la gersdorffite et la cobaltite enrichies en arsenic, tandis que les diarséniures vont de la rammelsbergite à la safflorite. La rammelsbergite possède une composition dans l'intervalle $\left(\mathrm{Ni}_{0.71-0.97} \mathrm{Co}_{0.02-0.29} \mathrm{Fe}_{0.00-0.02}\right) \mathrm{As}_{1.75-2.01} \mathrm{~S}_{0.00-0.26}$, et dépasse ainsi la valeur théorique attribuée par Yund (1962), $\left(\mathrm{As}_{1.93} \mathrm{~S}_{0.07}\right)$. Nous avons trouvé deux générations de compositions faisant partie de la série gersdorffite-cobaltite, comme en témoignent les attributs texturaux et la composition chimique; la première, $\left(\mathrm{Co}_{0.10-0.77} \mathrm{Ni}_{0.07-0.80} \mathrm{Fe}_{0.03-0.58}\right) \mathrm{As}_{0.95-}$ ${ }_{1.27} \mathrm{~S}_{0.75-1.06}$, fait preuve d'une corrélation positive entre les teneurs en $\mathrm{S}$ et $\mathrm{Fe}$, et en Co et $\mathrm{As}$, tandis que la seconde, $\left(\mathrm{Co}_{0.02-}\right.$

$\S \quad$ E-mail address: fanlo@unizar.es 
$\left.{ }_{0.91} \mathrm{Ni}_{0.02-0.96} \mathrm{Fe}_{0.00-0.28}\right) \mathrm{As}_{0.99-1.38} \mathrm{~S}_{0.67-1.01}$, possède une étendue considérable du champ de compositions dans la région pauvre en $\mathrm{Fe}$ du système CoAsS-NiAsS-FeAsS, montrant ainsi un remplacement important du Co pour le $\mathrm{Ni}$, avec une corrélation positive entre les teneurs en As et en Ni. La gersdorffite riche en arsenic montre en fait une variabilité importante de As et de S, sur l'intervalle $\left(\mathrm{Ni}_{0.65-0.98} \mathrm{Co}_{0.02-0.27} \mathrm{Fe}_{0.00-0.06}\right) \mathrm{As}_{1.37-1.83} \mathrm{~S}_{0.22-0.64}$, dépassant ainsi la valeur expérimentale proposée par Yund (1962), $\mathrm{NiAs}_{1.77} \mathrm{~S}_{0.23}$. La présence de l'alloclasite et l'étendue du phénomène de solutions solides des séries cobaltite-gersdorffite font penser que l'assemblage principal des minéraux du gisement de San Juan de Plan s'est formé à une température élevée, possiblement $600^{\circ} \mathrm{C}$. Des données géothermométriques préliminaires fondées sur le graphite concordent avec une telle température élevée.

(Traduit par la Rédaction)

Mots-clés: sulfarséniures de Ni-Co-Fe, solution solide gersdorffite-cobaltite, alloclasite, gersdorffite riche en arsenic, rammelsbergite, skutterudite, tracés de composition, San Juan de Plan, Espagne.

\section{INTRODUCTION}

We present here a detailed mineralogical and microanalytical study of sulfarsenides, diarsenides and triarsenides from the San Juan de Plan deposit, central Pyrenees, Spain. We wish to establish the different paragenetic assemblages in order to understand the evolution of the ore-forming process. These assemblages include, among other sulfarsenides and diarsenides, alloclasite, members of the gersdorffite-cobaltite solidsolution series, and As-rich gersdorffite coexisting with rammelsbergite. According to the experimental results of Yund (1962), Klemm (1965b), Maurel \& Picot (1974) and Hem \& Makovicky (2004), these assemblages must have formed at temperatures above $650-600^{\circ} \mathrm{C}$.

\section{BACKGROUND INFORMATION}

Compositional data reported in the literature, experimental determinations and theoretical considerations on phase relations in the system $\mathrm{Co}-\mathrm{Ni}-\mathrm{Fe}-\mathrm{As}-\mathrm{S}$ indicate the existence of the following features. 1) There is complete miscibility between gersdorffite (NiAsS) and cobaltite (CoAsS) above $550^{\circ} \mathrm{C}$. Conversely, the miscibility between arsenopyrite (FeAsS) and gersdorffitecobaltite solid-solution series is limited. The solvus reported by Klemm (1965b) in the system CoAsS$\mathrm{NiAsS}-\mathrm{Fe} A s \mathrm{~S}$ at temperatures between $650^{\circ}$ and $300^{\circ} \mathrm{C}$ has been used as a geothermometric indicator (e.g., Misra \& Fleet 1975, Oen et al. 1984, Gervilla \& Rønsbo 1992, Gervilla et al. 1996, 1998, Hem et al. 2001), in some cases yielding contradictory temperatures. 2) Limited miscibility exists between gersdorffite (cubic phase) and rammelsbergite (orthorhombic phase, $\mathrm{NiAs}_{2}$ ) along the rammelsbergite-vaesite $\left(\mathrm{NiS}_{2}\right)$ join. According to Yund (1962), the maximum As content of gersdorffite corresponds to the formula $\mathrm{NiAs}_{1.77} \mathrm{~S}_{0.23}$, and the maximum S content of rammelsbergite is $1.1 \pm 0.1 \mathrm{wt} \%$ at $700^{\circ} \mathrm{C}$. Nevertheless, a complete range of compositions between gersdorffite (NiAsS) and krutovite (cubic phase, $\mathrm{NiAs}_{2}$ ) has been reported by Spiridonov \& Chvileva (1995). 3) There is a small but real deficiency in arsenic in synthetic skutterudite, which also exists in natural skutterudite (Roseboom 1962, Nickel 1970). Recently, Hem \& Mackovicky (2004) have reported synthetic skutterudite with a $M e: X$ ratio from 1:2.84 to 1:3.02.

Hem \& Makovicky (2004) investigated the phase relations in the As-rich regions of the $(\mathrm{Fe}, \mathrm{Ni}, \mathrm{Co})(\mathrm{As}, \mathrm{S})_{2}$ prism at $650^{\circ}$ and $500^{\circ} \mathrm{C}$. They found that most of the phases exhibit extensive solid-solution at $650^{\circ} \mathrm{C}$, both in the systems $\mathrm{Fe}-\mathrm{Ni}-\mathrm{Co}$ and As-S. These authors confirmed the existence of complete solid-solution between gersdorffite and cobaltite at $650^{\circ} \mathrm{C}$ and showed the existence of complete solid-solutions among safflorite, alloclasite $[(\mathrm{Co}, \mathrm{Fe}) \mathrm{AsS}]$, skutterudite and their respective $\left(\mathrm{Fe}_{0.5} \mathrm{Ni}_{0.5}\right)$ analogues. However, these solid-solutions are more restricted with respect to $\mathrm{Fe}-\mathrm{Co}-\mathrm{Ni}$ at $500^{\circ} \mathrm{C}$, but they still show a large variation in As-S. They also found a nickel diarsenide containing up to 1 at.\% $\mathrm{Co}$ or $\mathrm{Fe}$ and $12-14$ at.\% $\mathrm{S}$ coexisting with gersdorffite. This phase, formed at $650^{\circ}$ and $500^{\circ} \mathrm{C}$, was identified as krutovite $\left(\mathrm{NiAs}_{2}\right)$. The phase in question contains much more sulfur than rammelsbergite formed at $600^{\circ}$ and $700^{\circ} \mathrm{C}$, as determined by Yund (1962). Krutovite formed at $500^{\circ} \mathrm{C}$ is notably richer in As than at $650^{\circ} \mathrm{C}$ and also coexists with gersdorffite. By powder X-ray diffraction (PXRD) and least-squares refinement of the unit cells of $(\mathrm{Fe}, \mathrm{Co})$-free gersdorffite and krutovite, Hem \& Makovicky (2004) gave the space group Pa3, with $a$ equal to $5.6917 \AA$ for gersdorffite and $5.723 \AA$ for the coexisting krutovite. This krutovite has compositions that partially overlap the gersdorffite field, as described by Yund (1962).

\section{The San Juan de Plan Deposit}

Mining activities in the San Juan de Plan area were first recorded in 1730 and concentrated on the cobalt ores. During the major period of mining activity from 1830 to 1870,35 t/yr were extracted with Co and $\mathrm{Ni}$ grades of 11 and $7 \%$, respectively. Since then, mining operations have been carried out at various scales. The last period of cobalt production ended in 1936. Later attempts to discover new resources failed. 
The ore is enclosed in a carbonate horizon at the top of a sequence of Silurian black shales. The roughly stratiform ore-bearing unit is $500 \mathrm{~m}$ long, with a maximum thickness of $2 \mathrm{~m}$, and is extensively converted to ankerite. The origin of this ankerite is epigenetic (Castroviejo \& Nodal 1985), on the basis of contact relationships between the ankerite and the precursor Silurian limestones. The ore-bearing ankerite horizon is controlled by east-west fractures and is located beneath Triassic red beds, which were deposited at the top of an erosional surface affecting the Paleozoic basement. This basement comprises a monotonous detrital sequence referred to as Cambrian-Ordovician, pre-Variscan augen gneisses and granitic gneisses, a Devonian sequence made up of limestones, calcareous slates and slates, and Carboniferous rocks composed of cherts, nodular limestones, dark slates and turbiditic sandstones. Granodioritic batholiths intrude the Paleozoic rocks. In places, ore deposits are hosted by porphyritic granitic dykes, which were emplaced along east-west fractures and the schistosity planes in black shales. The hydrothermal fluids promoted alteration of the dyke rocks (white mica and chlorite), the conversion of limestones to ankerite, and mineralization of replaced units.

Ore minerals typically occur as decimetric pockets, disseminations and veinlets locally linked to stylolitic seams rich in organic matter. Microscopic examinations confirm that the ore minerals are contemporaneous or rarely postdate ankerite formation. The ankerite and the ore minerals thus originated from the same process.

\section{Mineralogy and Petrography}

Mineral assemblages found in the San Juan de Plan deposit contain Co-Ni-Fe sulfarsenides, diarsenides and triarsenides, along with pyrite, arsenopyrite, nickeline, chalcopyrite, marcasite, native bismuth and bismuthinite. The gangue minerals mostly consist of ankerite, dolomite, and calcite. The more significant textural features of the San Juan de Plan deposit are replacements and overgrowths, as evidenced by large variations in $\mathrm{Fe}-$ $\mathrm{Co}-\mathrm{Ni}$ and As-S proportions in the zoned sulfarsenides.

Six stages of mineral deposition can be distinguished on the basis of the mineral association: Stage I: pyrite I, arsenopyrite, and scarce nickeline; Stage II: Co triarsenides (skutterudite I), and diarsenides (safflorite); Stage III: Co-Ni-Fe sulfarsenides: alloclasite and gersdorffite-cobaltite solid solution $\left(\mathrm{GC}_{\mathrm{ss}} \mathrm{I}\right)$; Stage IV: $\mathrm{Ni}-\mathrm{Co}$ sulfarsenides: gersdorffite-cobaltite solid solution $\left(\mathrm{GC}_{\mathrm{ss}} \mathrm{II}\right)$, arsenic-rich gersdorffite (As-Gdf) and $\mathrm{Ni}$ diarsenides (rammelsbergite and krutovite?); Stage V: Co triarsenides (skutterudite II); Stage VI: cobaltite, bismuthinite, pyrite II, chalcopyrite, marcasite and native bismuth (Fig. 1).

Stage I consists of euhedral pyrite I and arsenopyrite. Pyrite crystals are fractured and brecciated along cleavages. Locally, pyrite crystals act as nuclei for the crystallization of zoned $\mathrm{GC}_{\mathrm{ss}} \mathrm{I}$ and As-Gdf crystals at the outer rim (Fig. 2A). Arsenopyrite occurs as discrete euhedral crystals, in some cases replaced by one or both generations of $\mathrm{GC}_{\mathrm{ss}}$ crystals or alloclasite masses (Fig. 2B). No zoned, hourglass or obvious disequilibrium textures were observed in the arsenopyrite. This stage closes with the crystallization of nickeline, which has only been found as remnants of resorption in skutterudite I (Fig. 2C).

Stage II is marked by a drop in sulfur fugacity and a strong increase in arsenic fugacity, corresponding to the crystallization of triarsenides and diarsenides of cobalt. Skutterudite I is a scarce mineral; it occurs as minute inclusions in $\mathrm{GC}_{\mathrm{ss}} \mathrm{II}$ and As-Gdf crystals (Fig. 2C), and as irregular masses or euhedral crystals partially replaced by alloclasite (Fig. 2D). Safflorite also is scarce; it occurs as small and irregular masses enclosed in and partly replaced by alloclasite or $\mathrm{GC}_{\mathrm{ss}} \mathrm{I}$ crystals (Fig. 2E).

During stage III, sulfur fugacity increased slightly (and arsenic fugacity decreased), as deduced from the crystallization of alloclasite and $\mathrm{GC}_{\mathrm{ss}} \mathrm{I}$. Alloclasite exhibits clear purple to mauve to greenish olive rotation tints and occurs as skeletal masses or, locally, as rhombshaped crystals (Fig. 2D). The alloclasite areas contain well-defined optical sectors with lamellae parallel to crystals faces. Some irregular masses of alloclasite enclose euhedral crystals of pyrite I and arsenopyrite. $\mathrm{GC}_{\mathrm{ss}}$ I crystals are present as skeletal and irregular masses, euhedral to subhedral single crystals, or massive aggregates. The skeletal and irregular masses partially replace alloclasite (Fig. 2E) and rhombs of arsenopyrite.

Stage IV is characterized by the simultaneous deposition of gersdorffite-cobaltite solid-solution series $\left(\mathrm{GC}_{\mathrm{ss}} \mathrm{II}\right)$ and arsenic-rich gersdorffite (As-Gdf). In fact, $\mathrm{GC}_{\mathrm{ss}}$ II overgrows As-Gdf crystals showing idiomorphic terminations (Fig. $2 \mathrm{~F}$ ) or vice versa; the idiomorphic $\mathrm{GC}_{\mathrm{ss}}$ II crystals, showing significant growthinduced zoning, very fine in scale, of regular thickness and showing small variations in the As: $\mathrm{S}$ ratio and $\mathrm{Ni}$ contents, are overgrown by As-Gdf crystals (Figs. 2C, G). In addition, As-Gdf, or locally $\mathrm{GC}_{\mathrm{ss}}$ II, overgrew (Figs. 2H, I) irregular patches or euhedral lath-shaped crystals of $\mathrm{GC}_{\mathrm{ss}} \mathrm{I}$, and replaced and filled small cracks in these crystals. Arsenic-rich gersdorffite (As-Gdf) is not invariably a homogeneous phase, as the crystals show irregular microscopic growth-bands (Fig. 2G) characterized by small variations in the As and $\mathrm{S}$ contents but with no variation in metal content. Likewise, transitions between $\mathrm{GC}_{\mathrm{ss}}$ I and $\mathrm{GC}_{\mathrm{ss}}$ II and between these latter minerals and As-Gdf crystals have been recognized. Stage IV ends with the crystallization of rammelsbergite, which exhibits complex twinning consisting of extremely fine lamellae, with at least two twin laws, and rotation tints rich in different shades of blue, which indicates that rammelsbergite may come from pre-existing cubic krutovite. Rammelsbergite shows various modes of occurrence: (1) aggregates of prismatic tabular grains, randomly oriented with typical polysynthetic twins and, in some cases, enclosing 


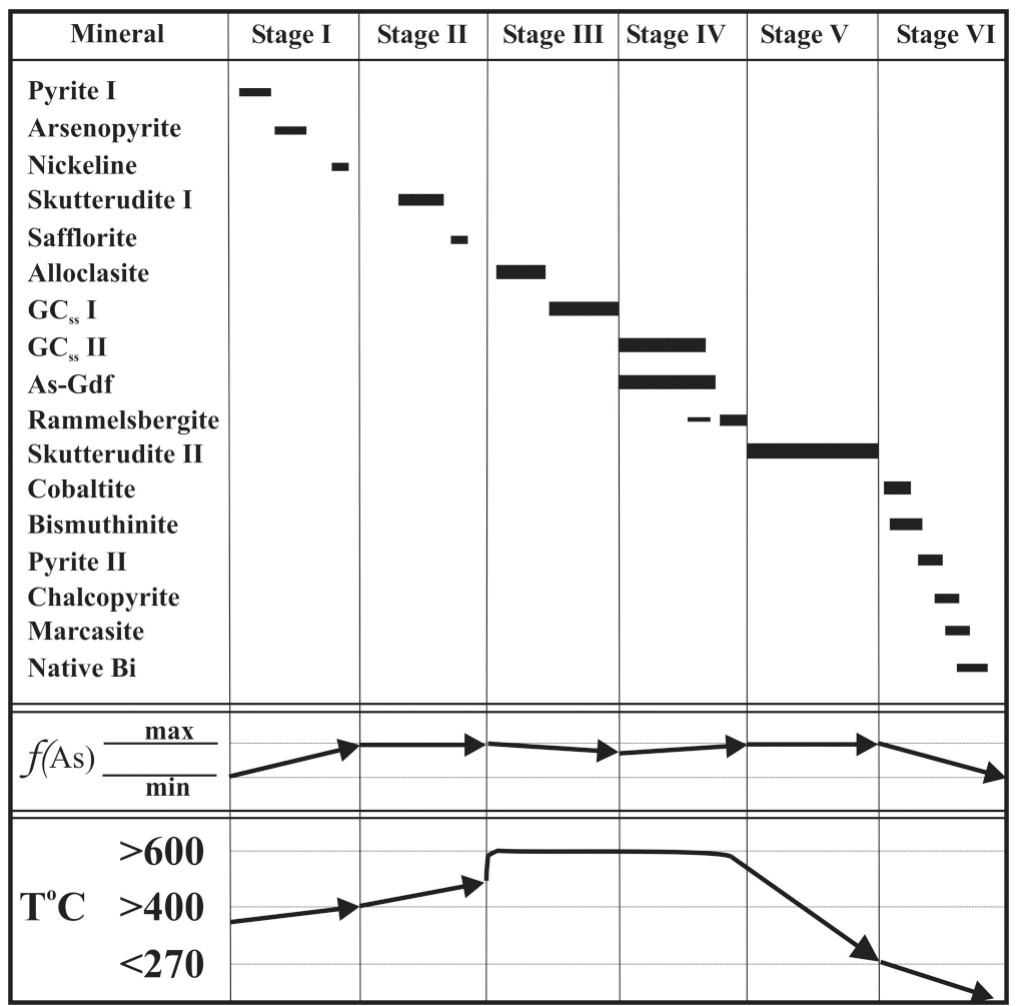

FIG. 1. Paragenetic sequence of the mineralization at San Juan de Plan. The estimated fugacity of arsenic and temperature during the precipitation of the different stages are shown. $\mathrm{GC}_{\mathrm{ss}} \mathrm{I}$, II: gersdorffite-cobaltite solid-solution series; As-Gdf: arsenic-rich gersdorffite.

euhedral crystals of skutterudite I, (2) skeletal masses, or small lath-shaped crystals with parallel alignment or pods in coarse idiomorphic crystals of skutterudite II (Figs. 2J, K), and (3) euhedral to subhedral single crystals partially replaced by crystals of skutterudite II.

Stage $V$ represents a further increase in the activity of arsenic, as indicated by the crystallization of skutterudite II. This mineral occurs as idiomorphic crystals replacing or overgrowing $\mathrm{GC}_{\mathrm{ss}} \mathrm{II}$, rammelsbergite and arsenic-rich gersdorffite (As-Gdf) crystals, which can be seen as islands along crystallographic directions in skutterudite II (Figs. 2J, K). Etching with $\mathrm{HNO}_{3}$ revealed zoning in skutterudite II without relation to compositional changes.

The mineral assemblage formed during stage VI consists of cobaltite, bismuthinite, pyrite II, native bismuth, chalcopyrite and marcasite. It shows characteristic textures of crystal growth in open spaces. Cobaltite precipitation is associated with bismuthinite and shows two distinctly different modes of occurrence: (1) subhedral grains isolated or forming aggregates of small size, with numerous micro-faults filled by bismuthinite; in some cases, both minerals show sharp contacts, which may suggest contemporaneous crystallization, and (2) irregular and small masses replacing both $\mathrm{GC}_{\mathrm{ss}} \mathrm{II}$ and As-Gdf crystals (Fig. 2F). Chalcopyrite fills cracks in skutterudite II, encloses $\mathrm{GC}_{\mathrm{ss}}$ I masses or where associated with bismuthinite, fills open spaces among $\mathrm{GC}_{\mathrm{ss}}$ II idiomorphic crystals. Pyrite II is partially transformed to marcasite and fills fractures and interstices in the mineral assemblage. Native bismuth is found as small inclusions within the earlier minerals or, as irregular masses filling the interstices and open spaces between rammelsbergite and skutterudite II.

\section{Mineral Compositions}

Most of the samples investigated in this study were obtained from the Asociación Mineralógica Aragonesa Museum and private collections; additional material was collected from dumps and accessible galleries. Thirty samples were studied by reflected-light microscopy, 
electron probe microanalysis, and X-ray diffraction using the powder method (PXRD).

Chemical compositions of the minerals investigated were determined by wavelength-dispersion electronprobe micro-analysis using a CAMECA SX-50 instrument at the University of Barcelona. The opaque minerals were analyzed for $\mathrm{Fe}, \mathrm{Co}, \mathrm{Ni}$, As and $\mathrm{S} ; \mathrm{Sb}$, $\mathrm{Cu}, \mathrm{Bi}, \mathrm{Zn}, \mathrm{Pt}$ and Pd were found to be below the detection limit or not detected. We monitored the peaks due to $\mathrm{Fe} K \alpha, \mathrm{Co} K \alpha, \mathrm{Ni} K \alpha, \mathrm{S} K \alpha, \mathrm{As} L \alpha, \mathrm{Sb} L \alpha, \mathrm{Cu} K \alpha$, $\mathrm{Zn} K \alpha, \mathrm{Bi} L \alpha$, and $\mathrm{Pb} M \alpha$. Operating conditions included an accelerating voltage of $20 \mathrm{kV}$ and a beam current of $20 \mathrm{nA}$. The counting times were $20 \mathrm{~s}$ on TAP/PET and $30 \mathrm{~s}$ on LiF crystals. The ZAF corrections were performed using the program supplied by CAMECA. Pyrite, $\mathrm{GaAs}, \mathrm{NiO}$, as well as pure Co metal, were used as primary standards. The maximum, minimum, mean and representative results of point analyses are given below in tables.

\section{Pyrite and arsenopyrite}

Pyrite has a nearly stoichiometric composition (Table 1): only minor As, Co and Ni contents were detected. Arsenopyrite (Table 1) contains some cobalt (up to $2.13 \mathrm{wt} \%$ ), but no nickel or antimony. The composition varies over the range $\left(\mathrm{Fe}_{0.94-1.02} \mathrm{Co}_{0.00-0.06}\right) \mathrm{As}_{0.88-}$ ${ }_{1.01} \mathrm{~S}_{0.99-1.11}$.

\section{Skutterudite}

Two generations of skutterudite can be distinguished on the basis of textural criteria (Fig. 1) and chemical composition (Table 2). Skutterudite I (stage II, Figs. 2C, D) is characterized by low $\mathrm{Ni}$ contents and the absence of Fe [ $\left.\left(\mathrm{Co}_{0.89-0.99} \mathrm{Ni}_{0.00-0.11}\right) \mathrm{As}_{2.84-2.99} \mathrm{~S}_{0.03-0.17}\right]$, whereas skutterudite II (stage V, Fig. 2J) is marked by a broader range of compositions $\left[\left(\mathrm{Co}_{0.50-090} \mathrm{Fe}_{0.05-0.22} \mathrm{Ni}_{0.05-}\right.\right.$ $\left.\left.{ }_{0.45}\right)^{A s} s_{2.75-3.01} \mathrm{~S}_{0.00-0.24}\right]$. Skutterudite II shows a substantial replacement of $\mathrm{Co}$ by $\mathrm{Ni}$ and $\mathrm{Fe}$, in the range of $1.35-11.35$ at.\% $\mathrm{Ni}$ and $1.36-5.59$ at.\% $\mathrm{Fe}$, and a limited replacement of As by S (0.00 to 6.12 at.\% S), but definitely higher than skutterudite I (from 0.70 to 4.14 at.\% S). In both generations of skutterudite, As is negatively correlated with $\mathrm{S}(\mathrm{R}=-0.97)$, whereas $\mathrm{Ni}$ and $\mathrm{Fe}$ show a weak positive correlation with As.

The compositions of skutterudite I and II plot within the solid-solution field (shaded area) defined by Roseboom (1962), Klemm (1965a) and Rosner (1970) in Co-Ni-Fe space (Fig. 3A). The arrows in Figure 3A show the compositional trends exhibited by the aforementioned generations of skutterudite. In skutterudite I, Co is replaced by $\mathrm{Ni}$ (Ni trend), whereas Co is replaced by a mixture of $\mathrm{Ni}$ and $\mathrm{Fe}(\mathrm{Ni} / \mathrm{Fe}$ trend) in skutterudite II. The regression line describing the composition of skutterudite $\mathrm{II}$ is $\mathrm{Ni}=0.65346-0.65319(\mathrm{Co}+\mathrm{Fe})$, with $\mathrm{R}=0.801$. The $\mathrm{Ni} / \mathrm{Fe}$ trend begins in the Co corner and cuts the $\mathrm{Ni}-\mathrm{Fe}$ join at $\mathrm{Ni}_{0.65} \mathrm{Fe}_{0.35}$.

In addition, the As-S plot (Fig. 3B), with a regression line of $\mathrm{As}=2.99699-0.96198 \mathrm{~S}, \mathrm{R}=0.969$ ) reveals that a significant part of the analyzed skutterudite does not show a deficiency in As (note that although only two points are over the $y$-axis indicating $\mathrm{S}=0$ and $\mathrm{As}=3.0$ atoms per formula unit, $a p f u$, they actually are representing 15 points). These analytical results constitute a discrepancy with the experimental work of Roseboom (1962), who said that natural skutterudite has a deficiency in arsenic.

\section{Diarsenides}

Electron-microprobe analyses show that safflorite has a nearly stoichiometric composition (Table 3 ): $\left(\mathrm{Co}_{0.89-0.97} \mathrm{Fe}_{0.02-0.10} \mathrm{Ni}_{0.00-0.02}\right) \mathrm{As}_{1.85-1.97} \mathrm{~S}_{0.04-0.16}$, and that As content is negatively correlated with $\mathrm{S}$ content. The regression line describing the relation between As

\begin{tabular}{|c|c|c|c|c|c|c|c|c|c|c|c|c|c|}
\hline & & \multicolumn{6}{|c|}{ Compositions (weight \%) } & \multicolumn{5}{|c|}{ Atoms per formula unit } & \multirow[b]{2}{*}{ As\# } \\
\hline & & $\mathrm{S}$ & As & $\mathrm{Fe}$ & $\mathrm{Co}$ & $\mathrm{Ni}$ & Total & $\mathrm{S}$ & As & $\mathrm{Fe}$ & $\mathrm{Co}$ & $\mathrm{Ni}$ & \\
\hline $\begin{array}{l}\text { Py } \\
n=27\end{array}$ & $\begin{array}{l}\min \\
\text { max } \\
\text { mean } \\
\text { C118 }\end{array}$ & $\begin{array}{l}50.95 \\
53.41 \\
52.58 \\
53.01\end{array}$ & $\begin{array}{l}0.01 \\
1.70 \\
0.28 \\
0.07\end{array}$ & $\begin{array}{l}44.70 \\
47.69 \\
46.30 \\
46.25\end{array}$ & $\begin{array}{l}0.04 \\
1.10 \\
0.16 \\
0.17\end{array}$ & $\begin{array}{l}\text { n.d. } \\
0.39 \\
0.10 \\
0.25\end{array}$ & $\begin{array}{r}99.25 \\
100.30 \\
99.79 \\
99.30\end{array}$ & $\begin{array}{l}1.95 \\
2.01 \\
1.99 \\
1.99\end{array}$ & $\begin{array}{l}0.00 \\
0.02 \\
0.00 \\
0.00\end{array}$ & $\begin{array}{l}0.98 \\
1.03 \\
1.01 \\
1.00\end{array}$ & $\begin{array}{l}0.00 \\
0.02 \\
0.00 \\
0.00\end{array}$ & $\begin{array}{l}0.00 \\
0.01 \\
0.00 \\
0.00\end{array}$ & $\begin{array}{l}- \\
- \\
-\end{array}$ \\
\hline $\begin{array}{l}\text { Apy } \\
n=17\end{array}$ & $\begin{array}{l}\min \\
\text { max } \\
\text { mean } \\
32 \\
35 \\
37 \\
38\end{array}$ & $\begin{array}{l}19.09 \\
22.39 \\
21.36 \\
22.04 \\
22.20 \\
21.27 \\
21.87\end{array}$ & $\begin{array}{l}41.23 \\
45.80 \\
42.81 \\
41.23 \\
41.86 \\
43.52 \\
42.48\end{array}$ & $\begin{array}{l}33.08 \\
35.42 \\
34.64 \\
34.73 \\
35.10 \\
35.03 \\
35.42\end{array}$ & $\begin{array}{l}0.02 \\
2.13 \\
0.34 \\
0.18 \\
0.39 \\
0.07 \\
0.02\end{array}$ & $\begin{array}{l}\text { n.d. } \\
0.28 \\
0.08 \\
0.11 \\
0.07 \\
0.02 \\
0.04\end{array}$ & $\begin{array}{r}98.80 \\
100.29 \\
99.65 \\
99.11 \\
100.29 \\
100.20 \\
99.96\end{array}$ & $\begin{array}{l}0.99 \\
1.11 \\
1.07 \\
1.11 \\
1.11 \\
1.06 \\
1.09\end{array}$ & $\begin{array}{l}0.88 \\
1.01 \\
0.92 \\
0.89 \\
0.89 \\
0.93 \\
0.91\end{array}$ & $\begin{array}{l}0.94 \\
1.02 \\
1.00 \\
1.00 \\
0.99 \\
1.01 \\
1.01\end{array}$ & $\begin{array}{l}0.00 \\
0.06 \\
0.01 \\
0.00 \\
0.02 \\
0.00 \\
0.00\end{array}$ & $\begin{array}{l}0.00 \\
0.00 \\
0.00 \\
0.00 \\
0.00 \\
0.00 \\
0.00\end{array}$ & $\begin{array}{l}0.44 \\
0.51 \\
0.46 \\
0.45 \\
0.44 \\
0.47 \\
0.46\end{array}$ \\
\hline
\end{tabular}

Representative results of micro-analyses are indicated in Figures $2 \mathrm{~A}$ and $2 \mathrm{~B}$, respectively. As\#: $\mathrm{As} /(\mathrm{As}+\mathrm{S})$. 

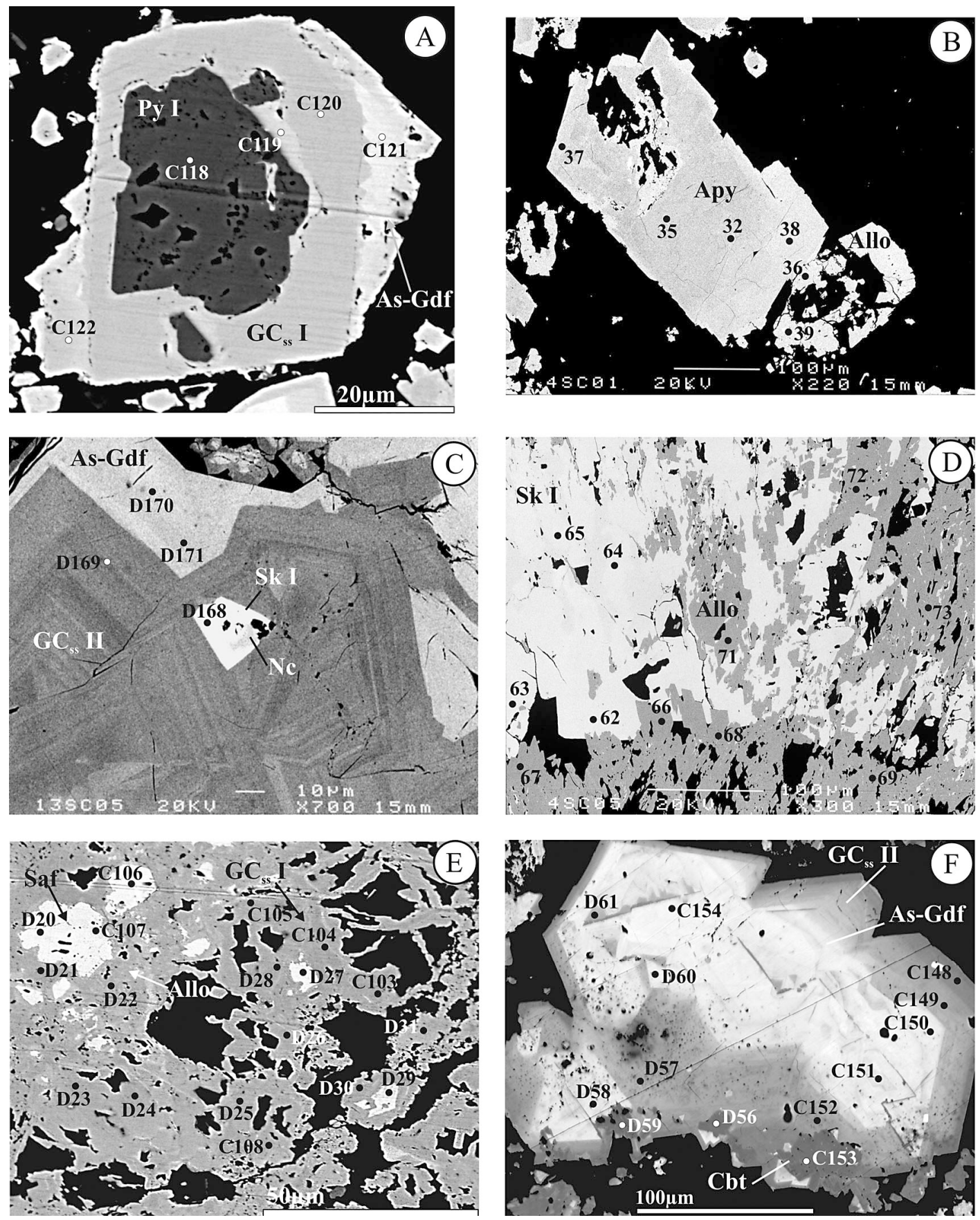

FIG. 2. Back-scattered electron images showing representative textures of the San Juan de Plan deposit. The numbers indicate compositions referenced in Tables 1 to 6. Symbols: Py I: pyrite from stage I; Apy: arsenopyrite; Nic: nickeline; Sk I: skutterudite from stage II; Sk II: skutterudite from stage V; Saf: safflorite; Ram: rammelsbergite; Allo: alloclasite; $\mathrm{GC}_{\mathrm{ss}} \mathrm{I}$ : gersdorffite-cobaltite solid-solution from stage III; $\mathrm{GC}_{\mathrm{ss}}$ II: gersdorffite-cobaltite solid-solution from stage IV; As-Gdf: arsenic-rich gersdorffite; Cbt: cobaltite from stage VI. (A). Pyrite I (Py I) enclosed by $\mathrm{GC}_{\mathrm{ss}} \mathrm{I}$ and As-Gdf crystals. (B). Euhedral crystal of arsenopyrite (Apy) partially replaced by alloclasite (Allo). (C). Minute inclusion of nickeline (Nc) hosted by skutterudite I (Sk I), which, in turn, is overgrown by $\mathrm{GC}_{\mathrm{ss}} \mathrm{II}$, showing complex zoning and growth history. The overgrowth 

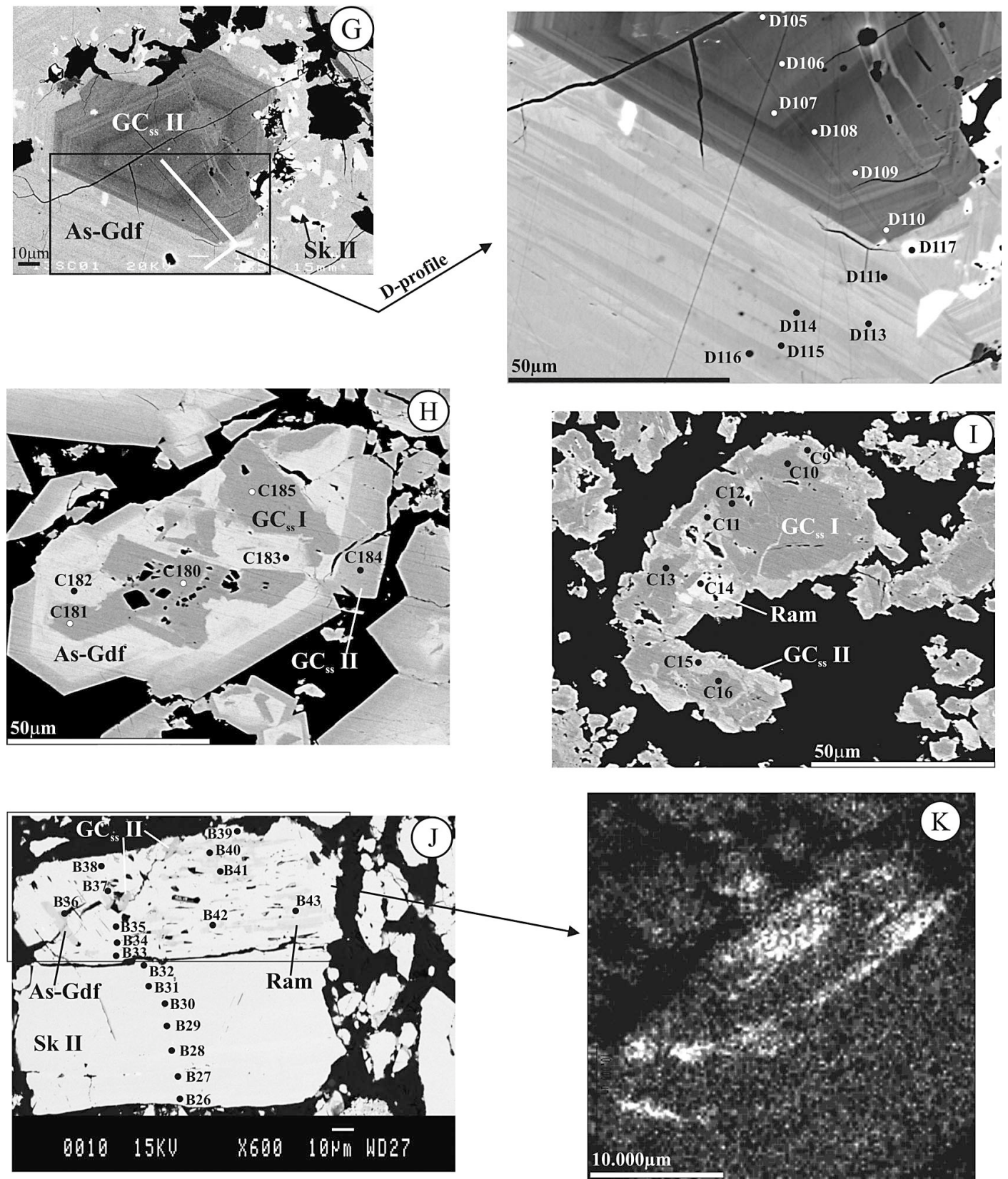

is composed of As-Gdf. (D). Alloclasite (Allo) replaces skutterudite I (Sk I) showing rhomb-shaped crystals. (E). Skeletal masses of safflorite (Saf) being replaced by alloclasite (Allo) or $\mathrm{GC}_{\mathrm{ss}} \mathrm{I}$. (F). Idiomorphic crystal of As-Gdf, partially replaced and overgrown by $\mathrm{GC}_{\mathrm{ss}} \mathrm{II}$, which displays oscillatory growth-zonation. Both minerals are replaced by cobaltite (Cbt). (G). Idiomorphic crystals of $\mathrm{GC}_{\mathrm{ss}}$ II showing very fine oscillatory growth-zonation, perfectly overgrown by As-Gdf, which shows irregular zonation; skutterudite II (Sk II) fills open spaces and partly replaces both minerals. (H). Irregular patches or lathshaped crystal of $\mathrm{GC}_{\mathrm{ss}}$ I overgrown by As-Gdf, which is, in turn, overgrown by $\mathrm{GC}_{\mathrm{ss}} \mathrm{II}$. (I). Small crystals of rammelsbergite (Ram) and irregular masses of $\mathrm{GC}_{\mathrm{ss}}$ I hosted by $\mathrm{GC}_{\mathrm{ss}}$ II. Note that $\mathrm{GC}_{\mathrm{ss}}$ II also fills cracks affecting $\mathrm{GC}_{\mathrm{ss}} \mathrm{I}$. (J). Idiomorphic skutterudite II (Sk II) replaces or overgrows $\mathrm{GC}_{\mathrm{ss}} \mathrm{II}, \mathrm{As}-\mathrm{Gdf}$ and rammelsbergite (Ram), which can be seen as islands along

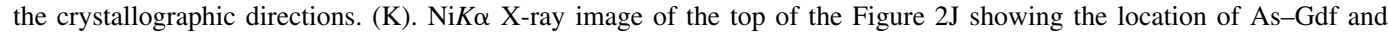
rammelsbergite crystals; the photomicrograph is rotated $30^{\circ}$ with respect to Figure 2J. 


\begin{tabular}{|c|c|c|c|c|c|c|c|c|c|c|c|c|c|}
\hline & & \multicolumn{6}{|c|}{ Compositions (weight \%) } & \multicolumn{5}{|c|}{ Atoms per formula unit } & \multirow[b]{2}{*}{ As\# } \\
\hline & & $\mathrm{S}$ & As & $\mathrm{Fe}$ & Co & $\mathrm{Ni}$ & Total & $\mathrm{S}$ & As & $\mathrm{Fe}$ & $\mathrm{Co}$ & $\mathrm{Ni}$ & \\
\hline $\begin{array}{l}\text { Sk I } \\
n=7\end{array}$ & $\begin{array}{l}\min \\
\max \\
\text { mean } \\
\text { D168 } \\
62 \\
63 \\
64 \\
65\end{array}$ & $\begin{array}{l}0.21 \\
1.9 \\
0.88 \\
0.51 \\
1.41 \\
0.62 \\
1.90 \\
1.13\end{array}$ & $\begin{array}{l}77.25 \\
79.86 \\
78.68 \\
79.33 \\
78.00 \\
78.80 \\
77.25 \\
78.20\end{array}$ & $\begin{array}{l}0.08 \\
0.27 \\
0.13 \\
0.09 \\
0.10 \\
0.17 \\
0.27 \\
0.14\end{array}$ & $\begin{array}{l}19.00 \\
20.92 \\
20.13 \\
19.00 \\
20.90 \\
20.70 \\
20.73 \\
20.90\end{array}$ & $\begin{array}{l}0.25 \\
2.42 \\
1.05 \\
2.42 \\
0.66 \\
0.25 \\
0.61 \\
0.26\end{array}$ & $\begin{array}{l}101.07 \\
101.62 \\
101.22 \\
101.35 \\
101.30 \\
101.17 \\
101.62 \\
101.03\end{array}$ & $\begin{array}{l}0.03 \\
0.17 \\
0.08 \\
0.06 \\
0.11 \\
0.06 \\
0.17 \\
0.11\end{array}$ & $\begin{array}{l}2.84 \\
2.99 \\
2.93 \\
2.94 \\
2.89 \\
2.96 \\
2.84 \\
2.91\end{array}$ & $\begin{array}{l}0.00 \\
0.00 \\
0.00 \\
0.00 \\
0.00 \\
0.00 \\
0.00 \\
0.00\end{array}$ & $\begin{array}{l}0.89 \\
0.99 \\
0.95 \\
0.89 \\
0.97 \\
0.99 \\
0.97 \\
0.98\end{array}$ & $\begin{array}{l}0.00 \\
0.11 \\
0.04 \\
0.11 \\
0.03 \\
0.00 \\
0.03 \\
0.00\end{array}$ & $\begin{array}{l}0.94 \\
0.99 \\
0.97 \\
0.98 \\
0.96 \\
0.98 \\
0.94 \\
0.96\end{array}$ \\
\hline $\begin{array}{l}\text { Sk II } \\
\mathrm{n}=84\end{array}$ & $\begin{array}{l}\min \\
\text { max } \\
\text { mean } \\
\text { D117 } \\
\text { B26 } \\
\text { B27 } \\
\text { B28 } \\
\text { B29 } \\
\text { B30 } \\
\text { B31 } \\
\text { B32 } \\
\text { B33 } \\
\text { B34 } \\
\text { B35 } \\
\text { B38 } \\
\text { B39 }\end{array}$ & $\begin{array}{l}0.03 \\
2.84 \\
1.02 \\
0.52 \\
2.24 \\
1.79 \\
2.09 \\
1.18 \\
1.80 \\
1.27 \\
1.30 \\
1.26 \\
0.11 \\
0.07 \\
0.05 \\
0.97\end{array}$ & $\begin{array}{l}75.87 \\
80.14 \\
78.32 \\
79.24 \\
76.6 \\
77.16 \\
76.75 \\
78.05 \\
77.22 \\
77.87 \\
77.48 \\
77.81 \\
79.59 \\
79.66 \\
79.55 \\
78.24\end{array}$ & $\begin{array}{l}0.84 \\
4.52 \\
2.25 \\
2.24 \\
1.15 \\
0.84 \\
1.40 \\
2.82 \\
1.59 \\
1.38 \\
1.54 \\
0.89 \\
2.71 \\
2.46 \\
3.66 \\
1.81\end{array}$ & $\begin{array}{l}10.50 \\
19.74 \\
14.30 \\
15.98 \\
16.67 \\
15.31 \\
16.13 \\
14.40 \\
15.48 \\
14.31 \\
15.64 \\
14.38 \\
13.15 \\
13.69 \\
12.03 \\
12.71\end{array}$ & $\begin{array}{l}0.89 \\
9.21 \\
4.41 \\
2.89 \\
3.54 \\
5.05 \\
3.86 \\
3.91 \\
3.92 \\
5.54 \\
3.65 \\
5.57 \\
4.84 \\
4.52 \\
5.22 \\
6.62\end{array}$ & $\begin{array}{r}99.39 \\
102.19 \\
100.60 \\
100.99 \\
100.47 \\
100.29 \\
100.30 \\
100.45 \\
100.16 \\
100.69 \\
99.75 \\
100.22 \\
100.56 \\
100.71 \\
100.66 \\
100.40\end{array}$ & $\begin{array}{l}0.00 \\
0.24 \\
0.09 \\
0.06 \\
0.19 \\
0.16 \\
0.19 \\
0.11 \\
0.17 \\
0.11 \\
0.11 \\
0.11 \\
0.00 \\
0.00 \\
0.00 \\
0.08\end{array}$ & $\begin{array}{l}2.75 \\
3.01 \\
2.91 \\
2.94 \\
2.81 \\
2.82 \\
2.79 \\
2.89 \\
2.84 \\
2.91 \\
2.88 \\
2.91 \\
3.01 \\
3.01 \\
2.99 \\
2.91\end{array}$ & $\begin{array}{l}0.05 \\
0.22 \\
0.11 \\
0.11 \\
0.06 \\
0.05 \\
0.08 \\
0.14 \\
0.08 \\
0.06 \\
0.08 \\
0.06 \\
0.14 \\
0.11 \\
0.02 \\
0.08\end{array}$ & $\begin{array}{l}0.50 \\
0.90 \\
0.68 \\
0.75 \\
0.77 \\
0.71 \\
0.74 \\
0.67 \\
0.72 \\
0.67 \\
0.76 \\
0.67 \\
0.62 \\
0.65 \\
0.56 \\
0.62\end{array}$ & $\begin{array}{l}0.05 \\
0.45 \\
0.21 \\
0.14 \\
0.17 \\
0.25 \\
0.19 \\
0.19 \\
0.19 \\
0.25 \\
0.17 \\
0.25 \\
0.23 \\
0.23 \\
0.25 \\
0.31\end{array}$ & $\begin{array}{l}0.92 \\
1.00 \\
0.97 \\
0.98 \\
0.94 \\
0.95 \\
0.94 \\
0.96 \\
0.94 \\
0.96 \\
0.96 \\
0.96 \\
1.00 \\
1.00 \\
1.00 \\
0.97\end{array}$ \\
\hline
\end{tabular}

Representative results of micro-analyses are indicated in Figures $2 \mathrm{D}, 2 \mathrm{G}$, and $2 \mathrm{~J} . \mathrm{As} \#$ : As/ $/(\mathrm{As}+\mathrm{S})$.

TABLE 3. STATISTICAL RESULTS OF ELECTRON-MICROPROBE ANALYSES OF SAFFLORITE (STAGE II) AND RAMMELSBERGITE (STAGE IV) FROM THE SAN JUAN DE PLAN DEPOSIT, CENTRAL PYRENEES, SPAIN

\begin{tabular}{|c|c|c|c|c|c|c|c|c|c|c|c|c|c|}
\hline & & \multicolumn{6}{|c|}{ Compositions (weight \%) } & \multicolumn{5}{|c|}{ Atoms per formula unit } & \multirow[b]{2}{*}{ Ast } \\
\hline & & $\mathrm{S}$ & As & $\mathrm{Fe}$ & $\mathrm{Co}$ & $\mathrm{Ni}$ & Total & $\mathrm{S}$ & As & $\mathrm{Fe}$ & $\mathrm{Co}$ & $\mathrm{Ni}$ & \\
\hline $\begin{array}{l}\text { Saf } \\
n=6\end{array}$ & $\begin{array}{l}\min \\
\text { max } \\
\text { mean } \\
\text { C106 } \\
\text { C107 } \\
\text { D20 } \\
\text { D27 } \\
\text { D29 }\end{array}$ & $\begin{array}{l}0.68 \\
2.44 \\
1.77 \\
1.99 \\
1.47 \\
2.44 \\
1.70 \\
2.34\end{array}$ & $\begin{array}{l}68.69 \\
71.13 \\
69.62 \\
69.84 \\
69.65 \\
68.83 \\
69.67 \\
68.69\end{array}$ & $\begin{array}{l}0.42 \\
2.97 \\
1.47 \\
1.44 \\
0.95 \\
1.13 \\
0.42 \\
2.97\end{array}$ & $\begin{array}{l}25.69 \\
27.96 \\
27.11 \\
27.47 \\
27.66 \\
27.96 \\
27.69 \\
25.69\end{array}$ & $\begin{array}{l}0.00 \\
0.71 \\
0.35 \\
0.57 \\
0.48 \\
0.00 \\
0.00 \\
0.35\end{array}$ & $\begin{array}{r}99.12 \\
101.68 \\
100.50 \\
101.63 \\
101.11 \\
99.12 \\
99.84 \\
100.36\end{array}$ & $\begin{array}{l}0.04 \\
0.16 \\
0.11 \\
0.12 \\
0.10 \\
0.16 \\
0.10 \\
0.14\end{array}$ & $\begin{array}{l}1.85 \\
1.97 \\
1.89 \\
1.86 \\
1.89 \\
1.85 \\
1.91 \\
1.85\end{array}$ & $\begin{array}{l}0.02 \\
0.10 \\
0.05 \\
0.06 \\
0.04 \\
0.04 \\
0.02 \\
0.10\end{array}$ & $\begin{array}{l}0.89 \\
0.97 \\
0.93 \\
0.94 \\
0.95 \\
0.95 \\
0.97 \\
0.89\end{array}$ & $\begin{array}{l}0.00 \\
0.02 \\
0.01 \\
0.02 \\
0.02 \\
0.00 \\
0.00 \\
0.02\end{array}$ & $\begin{array}{l}0.92 \\
0.98 \\
0.95 \\
0.94 \\
0.95 \\
0.92 \\
0.95 \\
0.93\end{array}$ \\
\hline $\begin{array}{l}\operatorname{Ram} \\
n=42\end{array}$ & $\begin{array}{l}\min \\
\text { max } \\
\text { mean } \\
\text { C14 } \\
\text { B37 } \\
\text { B41 } \\
\text { B42 } \\
\text { B43 }\end{array}$ & $\begin{array}{l}0.08 \\
4.22 \\
1.96 \\
2.17 \\
2.85 \\
2.53 \\
4.06 \\
2.90\end{array}$ & $\begin{array}{l}66.30 \\
72.31 \\
69.65 \\
68.96 \\
68.95 \\
68.63 \\
66.28 \\
69.36\end{array}$ & $\begin{array}{l}0.01 \\
0.61 \\
0.17 \\
0.59 \\
0.45 \\
0.13 \\
0.21 \\
0.40\end{array}$ & $\begin{array}{l}0.73 \\
8.41 \\
2.34 \\
0.76 \\
1.82 \\
2.45 \\
1.97 \\
4.37\end{array}$ & $\begin{array}{l}19.73 \\
28.70 \\
26.03 \\
26.70 \\
25.80 \\
26.00 \\
26.73 \\
22.68\end{array}$ & $\begin{array}{c}99.11 \\
101.59 \\
100.4 \\
99.28 \\
99.97 \\
99.93 \\
99.44 \\
99.81\end{array}$ & $\begin{array}{l}0.00 \\
0.26 \\
0.12 \\
0.14 \\
0.18 \\
0.16 \\
0.26 \\
0.18\end{array}$ & $\begin{array}{l}1.75 \\
2.01 \\
1.89 \\
1.88 \\
1.85 \\
1.86 \\
1.76 \\
1.87\end{array}$ & $\begin{array}{l}0.00 \\
0.02 \\
0.00 \\
0.02 \\
0.02 \\
0.00 \\
0.00 \\
0.02\end{array}$ & $\begin{array}{l}0.02 \\
0.29 \\
0.08 \\
0.02 \\
0.06 \\
0.08 \\
0.06 \\
0.14\end{array}$ & $\begin{array}{l}0.71 \\
0.96 \\
0.90 \\
0.94 \\
0.89 \\
0.89 \\
0.92 \\
0.79\end{array}$ & $\begin{array}{l}0.87 \\
1.00 \\
0.94 \\
0.93 \\
0.92 \\
0.92 \\
0.87 \\
0.91\end{array}$ \\
\hline
\end{tabular}

Representative results of micro-analyses are indicated in Figures 2D, 2I, and 2J. As : As/(As + S).

and $\mathrm{S}$ is $\mathrm{As}=2.008-1.081 \mathrm{~S}(\mathrm{R}=-0.96) . \mathrm{Fe}$ and $\mathrm{Ni}$ contents are negatively correlated with Co content $(\mathrm{R}=$ -0.94 and -0.66 , respectively). The composition of the pure cobalt end-member in the system $\mathrm{CoAs}_{2}-\mathrm{NiAs}_{2}-$ $\mathrm{FeAs}_{2}$ (Fig. 4) has only been reported previously in natural assemblages by Radcliffe \& Berry (1968).
Regarding rammelsbergite, the electron-microprobe data (Table 3) show a composition within the range $\left(\mathrm{Ni}_{0.71-0.96} \mathrm{Co}_{0.02-0.29} \mathrm{Fe}_{0.00-0.02}\right) \mathrm{As}_{1.75-2.01} \mathrm{~S}_{0.00-0.26}$. There is a strong negative correlation between As and $\mathrm{S}$, with a regression line $\mathrm{As}=2.005-0.058 \mathrm{~S}(\mathrm{R}=-0.99)$. 


\section{Sulfarsenides}

Sulfarsenides, including alloclasite, members of the gersdorffite-cobaltite solid-solution series, arsenic-rich gersdorffite and cobaltite, are the most abundant ore minerals and were formed during stage III and stage IV, except for cobaltite, which was precipitated in stage VI (Fig. 1). The electron-microprobe data show that the compositional variability of alloclasite, expressed as $\left(\mathrm{Co}_{0.52-0.96} \mathrm{Ni}_{0.00-0.27} \mathrm{Fe}_{0.02-0.39}\right) \mathrm{As}_{0.99-1.14} \mathrm{~S}_{0.88-1.02}$, exhibits a well-defined trend (Fig. 5A) characterized by the substitution of $\mathrm{Co}$ by a mixture of $\mathrm{Ni}$ and $\mathrm{Fe}$ (Table 4). According to Hem et al. (2001), this Ni/Fe trend is indicative of a metal-rich genetic environment. The As:S ratio ranges from 0.98 to 1.30 (1.07 \pm 0.06$)$,
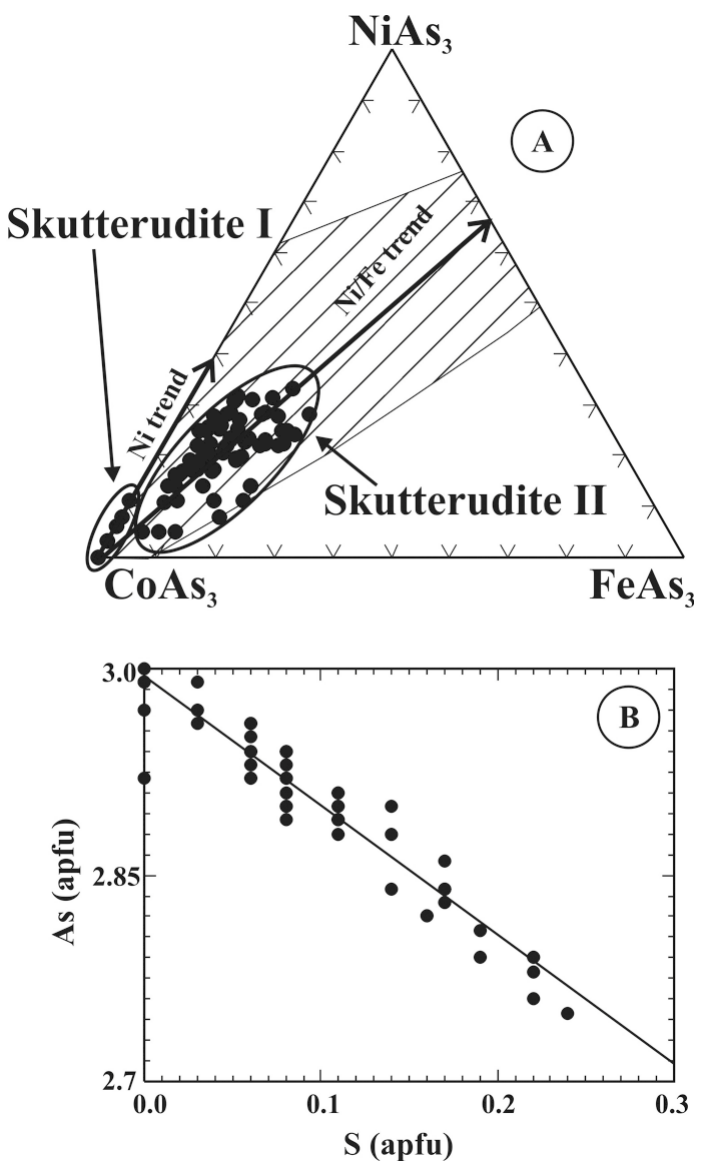

FIG. 3. A. Plot of skutterudite I and skutterudite II compositions (in mol.\%) in the system $\mathrm{FeAs}_{3}-\mathrm{CoAs}_{3}-\mathrm{NiAs}_{3}$. The arrows indicate the trend for each type of skutterudite, and the shaded area represents the solid-solution field defined by Roseboom (1962). B. Binary plot of As versus S in atoms per formula unit. and the regression line is $\mathrm{Ni}=0.744-0.792 \mathrm{Co}(\mathrm{R}=$ $0.91)$.

The chemical composition of $\mathrm{GC}_{\mathrm{ss}}$ I crystals (Table 5) $\left(\mathrm{Co}_{0.10-0.74} \mathrm{Ni}_{0.07-0.80} \mathrm{Fe}_{0.03-0.58}\right) \mathrm{As}_{0.95-1.20} \mathrm{~S}_{0.83-}$ 1.06 , shows an As:S ratio varying from 0.90 to 1.45 (1.07 \pm 0.11 ), and a strong negative correlation between ( $\mathrm{Fe}$ $+\mathrm{Ni})$ and $\mathrm{Co}(\mathrm{R}=-0.99)$, indicative of their mutual substitution. Consequently, $\mathrm{GC}_{\mathrm{ss}} \mathrm{I}$ crystals display the same type of compositional trend as alloclasite, i.e., a $\mathrm{Ni} / \mathrm{Fe}$ trend characterized by the substitution of Co by a mixture of $\mathrm{Ni}$ and $\mathrm{Fe}$ (Fig. 5A), with the regression line $\mathrm{Ni}=0.6013-0.56526 \mathrm{Co}(\mathrm{R}=0.72)$. The compositional fields of alloclasite and $\mathrm{GC}_{\mathrm{ss}} \mathrm{I}$ and the textural relationships (Fig. 2E) suggest that alloclasite was replaced by $\mathrm{GC}_{\mathrm{ss}} \mathrm{I}$ in a metal-rich environment (Hem et al. 2001) characterized by an increase in $\mathrm{Fe}$ and $\mathrm{Ni}$ concentration with a concomitant decrease in As concentration. Moreover, the overlap between the compositional fields of alloclasite and $\mathrm{GC}_{\mathrm{ss}}$ I may be interpreted as a compositional continuum between alloclasite and the $\mathrm{GC}_{\mathrm{ss}}$ I phase as Ni activity increased. The increase in Ni contents started with the crystallization of alloclasite instead of safflorite (Fig. 2E).

The phase $\mathrm{GC}_{\mathrm{ss}}$ II $\left(\mathrm{Co}_{0.02-0.81} \mathrm{Ni}_{0.10-0.96} \mathrm{Fe}_{0.00-}\right.$ $\left.{ }_{0.28}\right)^{\mathrm{As}_{1.01-1.36}} \mathrm{~S}_{0.67-1.01}$ exhibits considerable compositional variability (Table 5) in the $\mathrm{Fe}$-poor region of the system CoAsS-NiAsS-FeAsS (Fig. 6A). The As:S ratio ranges from 1.00 to $2.03(1.43 \pm 0.31)$. This variability is directly related to the $\mathrm{Ni}$ contents, as there is a posi-

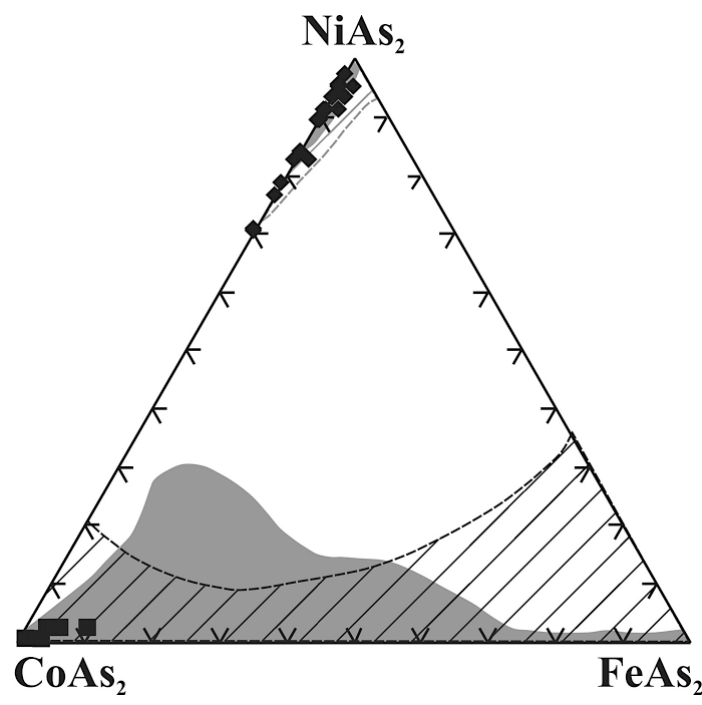

FIG. 4. Plot of diarsenide compositions in the system $\mathrm{CoAs}_{2}$ $\mathrm{NiAs}_{2}-\mathrm{FeAs}_{2}$. Black squares: safflorite; black diamonds: rammelsbergite. The compositional limits of natural $(\mathrm{Ni}$, $\mathrm{Fe}, \mathrm{Co}$ ) diarsenides according to Roseboom (1963) (dashed area) and Radcliffe \& Berry (1968) (full area) also are shown. 


\begin{tabular}{|c|c|c|c|c|c|c|c|c|c|c|c|c|c|}
\hline & & \multicolumn{6}{|c|}{ Compositions (weight \%) } & \multicolumn{5}{|c|}{ Atoms per formula unit } & \multirow[b]{2}{*}{ As\# } \\
\hline & & $\mathrm{S}$ & As & $\mathrm{Fe}$ & $\mathrm{Co}$ & $\mathrm{Ni}$ & Total & $\mathrm{S}$ & As & $\mathrm{Fe}$ & $\mathrm{Co}$ & $\mathrm{Ni}$ & \\
\hline $\begin{array}{l}\text { Alo } \\
n=81\end{array}$ & $\begin{array}{l}\min \\
\text { max } \\
\text { mean } \\
36 \\
39 \\
66 \\
67 \\
68 \\
69 \\
71 \\
72 \\
73 \\
C 103 \\
\text { C108 } \\
\text { D21 } \\
\text { D22 } \\
\text { D23 } \\
\text { D24 } \\
\text { D28 } \\
\text { D30 }\end{array}$ & $\begin{array}{l}16.37 \\
19.73 \\
18.63 \\
17.91 \\
18.44 \\
18.21 \\
17.36 \\
18.65 \\
18.24 \\
18.58 \\
18.12 \\
17.40 \\
18.55 \\
18.85 \\
19.22 \\
17.28 \\
18.12 \\
18.12 \\
17.05 \\
16.37\end{array}$ & $\begin{array}{l}45.02 \\
49.74 \\
46.42 \\
46.47 \\
45.80 \\
46.16 \\
47.59 \\
45.96 \\
45.49 \\
45.30 \\
45.62 \\
46.93 \\
46.65 \\
46.56 \\
46.46 \\
48.25 \\
46.70 \\
47.37 \\
49.39 \\
49.74\end{array}$ & $\begin{array}{r}0.32 \\
12.80 \\
2.80 \\
6.39 \\
5.08 \\
1.92 \\
1.58 \\
4.48 \\
3.90 \\
12.80 \\
4.76 \\
5.90 \\
1.86 \\
1.42 \\
1.88 \\
1.58 \\
1.96 \\
1.77 \\
1.02 \\
1.30\end{array}$ & $\begin{array}{l}17.99 \\
34.32 \\
28.65 \\
24.80 \\
25.83 \\
30.49 \\
30.73 \\
27.99 \\
28.82 \\
17.99 \\
27.59 \\
25.98 \\
27.67 \\
26.98 \\
26.66 \\
26.36 \\
25.54 \\
25.09 \\
25.64 \\
30.44\end{array}$ & $\begin{array}{l}0.00 \\
9.43 \\
3.43 \\
3.65 \\
4.33 \\
2.70 \\
2.95 \\
2.76 \\
2.29 \\
3.66 \\
2.64 \\
2.70 \\
4.87 \\
6.01 \\
5.54 \\
6.16 \\
6.08 \\
7.12 \\
6.84 \\
1.87\end{array}$ & $\begin{array}{r}98.53 \\
101.47 \\
100.28 \\
100.09 \\
99.59 \\
99.77 \\
100.35 \\
101.01 \\
98.91 \\
98.60 \\
98.94 \\
99.60 \\
99.75 \\
99.99 \\
99.12 \\
100.08 \\
98.93 \\
99.66 \\
100.00 \\
100.03\end{array}$ & $\begin{array}{l}0.88 \\
1.02 \\
0.97 \\
0.95 \\
0.97 \\
0.96 \\
0.91 \\
0.97 \\
0.96 \\
0.98 \\
0.96 \\
0.92 \\
0.98 \\
0.98 \\
0.97 \\
0.92 \\
0.97 \\
0.96 \\
0.90 \\
0.88\end{array}$ & $\begin{array}{l}0.99 \\
1.14 \\
1.04 \\
1.05 \\
1.02 \\
1.04 \\
1.08 \\
1.02 \\
1.03 \\
1.01 \\
1.03 \\
1.07 \\
1.04 \\
1.03 \\
1.06 \\
1.09 \\
1.06 \\
1.06 \\
1.12 \\
1.14\end{array}$ & $\begin{array}{l}0.02 \\
0.39 \\
0.08 \\
0.19 \\
0.15 \\
0.05 \\
0.05 \\
0.13 \\
0.12 \\
0.39 \\
0.15 \\
0.19 \\
0.05 \\
0.05 \\
0.05 \\
0.05 \\
0.07 \\
0.05 \\
0.03 \\
0.03\end{array}$ & $\begin{array}{l}0.52 \\
0.96 \\
0.81 \\
0.71 \\
0.74 \\
0.87 \\
0.88 \\
0.79 \\
0.83 \\
0.52 \\
0.79 \\
0.75 \\
0.79 \\
0.77 \\
0.77 \\
0.77 \\
0.83 \\
0.72 \\
0.75 \\
0.90\end{array}$ & $\begin{array}{l}0.00 \\
0.27 \\
0.10 \\
0.10 \\
0.12 \\
0.08 \\
0.08 \\
0.08 \\
0.07 \\
0.10 \\
0.07 \\
0.08 \\
0.13 \\
0.17 \\
0.15 \\
0.17 \\
0.17 \\
0.20 \\
0.20 \\
0.05\end{array}$ & $\begin{array}{l}0.50 \\
0.56 \\
0.52 \\
0.53 \\
0.51 \\
0.52 \\
0.54 \\
0.51 \\
0.52 \\
0.51 \\
0.52 \\
0.54 \\
0.51 \\
0.51 \\
0.52 \\
0.54 \\
0.52 \\
0.52 \\
0.55 \\
0.56\end{array}$ \\
\hline $\begin{array}{l}\mathrm{Cbt} \\
n=18\end{array}$ & $\begin{array}{l}\min \\
\text { max } \\
\text { mean } \\
\text { C153 } \\
\text { D56 } \\
\text { D59 }\end{array}$ & $\begin{array}{l}17.52 \\
19.70 \\
18.96 \\
19.15 \\
19.29 \\
19.22\end{array}$ & $\begin{array}{l}41.74 \\
48.13 \\
45.88 \\
45.94 \\
46.50 \\
45.82\end{array}$ & $\begin{array}{l}1.02 \\
4.19 \\
2.38 \\
2.66 \\
2.25 \\
1.29\end{array}$ & $\begin{array}{l}23.42 \\
33.10 \\
29.94 \\
32.02 \\
32.42 \\
33.10\end{array}$ & $\begin{array}{l}0.09 \\
9.26 \\
2.57 \\
0.45 \\
0.09 \\
0.41\end{array}$ & $\begin{array}{r}99.33 \\
101.34 \\
100.60 \\
100.56 \\
100.66 \\
100.07\end{array}$ & $\begin{array}{l}0.93 \\
1.01 \\
0.99 \\
0.99 \\
0.99 \\
1.00\end{array}$ & $\begin{array}{l}0.99 \\
1.08 \\
1.02 \\
1.01 \\
1.03 \\
1.02\end{array}$ & $\begin{array}{l}0.03 \\
0.13 \\
0.07 \\
0.08 \\
0.07 \\
0.03\end{array}$ & $\begin{array}{l}0.68 \\
0.93 \\
0.85 \\
0.90 \\
0.91 \\
0.93\end{array}$ & $\begin{array}{l}0.00 \\
0.27 \\
0.08 \\
0.02 \\
0.00 \\
0.02\end{array}$ & $\begin{array}{l}0.50 \\
0.54 \\
0.51 \\
0.51 \\
0.51 \\
0.50\end{array}$ \\
\hline
\end{tabular}

Representative results of micro-analyses are indicated in Figures 2D, 2E, and 2F. As\#: As/(As + S).

tive correlation between $\mathrm{As}$ and $\mathrm{Ni}(\mathrm{R}=0.70)$. As a whole, the composition of $\mathrm{GC}_{\mathrm{ss}}$ II defines a chemical trend characterized by the substitution of $\mathrm{Co}$ by $\mathrm{Ni}$ which, according to Hem et al. (2001), is consistent with a significant increase in the As content. Phase $\mathrm{GC}_{\mathrm{ss}} \mathrm{II}$ is overgrown by As-rich gersdorffite, and this was followed by the crystallization of rammelsbergite and skutterudite.

The arsenic-rich gersdorffite (Table 6) displays a narrow range in metal contents (Fig. 6A) and a broad variability in $\mathrm{As}$ and $\mathrm{S}$ contents: $\left(\mathrm{Ni}_{065-0.98} \mathrm{Co}_{0.02-}\right.$ $\left.{ }_{0.27} \mathrm{Fe}_{0.00-0.06}\right) \mathrm{As}_{1.28-1.75} \mathrm{~S}_{0.28-0.77}$. It shows a negative correlation between the sum $\mathrm{Co}+\mathrm{Fe}$ and $\mathrm{Ni}$ content $(\mathrm{Co} / \mathrm{Fe}$ trend) with a regression line $\mathrm{Ni}=0.988-1.113$ $(\mathrm{Co}+\mathrm{Fe}), \mathrm{R}=-0.93)$. The assemblage in which this trend is found suggests an environment with a high fugacity of arsenic, as shown by Hem et al. (2001) in a magmatic deposit associated with the Ronda peridotite (Spain). There is no correlation among cations and anions, and the As:S ratio is very variable, suggesting that As content is independent of the metal content.

The textural relations among $\mathrm{GC}_{\mathrm{ss}} \mathrm{I}, \mathrm{GC}_{\mathrm{ss}} \mathrm{II}$ and AsGdf shown in Figure 2H, along with their compositions in the system NiAsS-CoAsS-FeAsS and the relation between As and Ni contents, have been plotted in Figures $6 \mathrm{~B}$ and $6 \mathrm{C}$. From $\mathrm{GC}_{\mathrm{ss}}$ I to As-Gdf, there is a significant increase in $\mathrm{Ni}$ and As contents. In Figures 6D and $6 \mathrm{E}$ (relating to Fig. 2G), the compositional varia- tion between $\mathrm{GC}_{\mathrm{ss}}$ II and As-Gdf (Tables 5, 6) is shown. Both crystals display identical Ni contents, but As contents are much higher in As-Gdf. $\mathrm{GC}_{\mathrm{ss}}$ II and As-Gdf crystals show fine oscillatory growth-zoning characterized by small variations in As and $\mathrm{S}$ contents.

Cobaltite, $\left(\mathrm{Co}_{0.68-0.93} \mathrm{Ni}_{0.00-0.27} \mathrm{Fe}_{0.03-0.12}\right) \mathrm{As}_{0.99-}$ ${ }_{1.08} \mathrm{~S}_{0.93-1.01}$, displays a narrower compositional range than alloclasite (Table 4). Electron-microprobe analyses show a small excess of As over S, which is in disagreement with the findings of Maurel \& Picot (1974). The amount of Co and the As:S ratio correlate negatively $(\mathrm{R}=-0.75)$.

\section{DisCUSSION}

Compositional trends and extent of solid solution in the system $\mathrm{Ni}-\mathrm{Co}-\mathrm{Fe}-\mathrm{As}-\mathrm{S}$

Mineralogical evidence suggests that the compositional trends exhibited by the $\mathrm{Ni}-\mathrm{Co}-\mathrm{Fe}$ phases found in the San Juan de Plan deposit, as well as the crystallization sequence, are linked to changes in activities of key elements in the mineralizing fluids. For example, the compositional variation in skutterudite shows that $\mathrm{Ni}$ and $\mathrm{Fe}$ substitute for Co in different proportions, depending on the paragenetic position of skutterudite (Fig. 3A). Skutterudite I, along with $\mathrm{GC}_{\mathrm{ss}}$ II (Fig. 6A), display a Ni-enrichment trend, marked by the replace- 
ment of $\mathrm{Co}$ for $\mathrm{Ni}$, which suggests high fugacities of the anion (As) (Hem et al. 2001). The sequential formation of $\mathrm{GC}_{\mathrm{ss}} \mathrm{II}, \mathrm{As}-\mathrm{Gdf}$, rammelsbergite and skutterudite II also show a crystallization trend characterized by the progressive increase in the As fugacity as a continuation of the $\mathrm{Ni}$ trend defined by $\mathrm{GC}_{\mathrm{ss}}$ II. In contrast, alloclasite, $\mathrm{GC}_{\mathrm{ss}}$ I and skutterudite II (Figs. 3A, 5A) display a $\mathrm{Ni} / \mathrm{Fe}$ trend, in which $\mathrm{Co}$ is replaced by a mixture of $\mathrm{Ni}$ and $\mathrm{Fe}$. The $\mathrm{Ni} / \mathrm{Fe}$ trend indicates that these phases crystallized in a metal-rich environment (Hem et al. 2001).

If we take into account that alloclasite is paragenetically earlier than $\mathrm{GC}_{\mathrm{ss}} \mathrm{I}$, an increase in $\mathrm{Ni}$ activity during stage III should inhibit the precipitation of alloclasite, favoring instead the crystallization of $\mathrm{GC}_{\mathrm{ss}}$ I. This inference is in agreement with the idea that alloclasite forms under high As fugacity and moderate Ni activity (Maurel \& Picot 1974). Where the activity of $\mathrm{Ni}$ increases, the deposition of alloclasite ceases, which favors the precipitation of $\mathrm{GC}_{\mathrm{ss}} \mathrm{I}$. The overlap between the compositional fields of alloclasite and $\mathrm{GC}_{\mathrm{ss}}$ $\mathrm{I}$, as well as their similar chemical trends, support this conclusion (Fig. 5A).

In comparison with available compositions of gersdorffite-cobaltite ${ }_{\mathrm{ss}}$ taken from the literature, our $\mathrm{GC}_{\mathrm{ss}}$ I data partly overlap those of Oen et al. (1971), Petruk et al. (1971), Gervilla \& Rønsbo (1992) and Gervilla et al. (1998). On the other hand, the data of Wagner \& Lorenz (2002) are similar to ours for $\mathrm{GC}_{\mathrm{ss}}$ II. Gersdorffite-cobaltite $_{\mathrm{ss}}$ from the San Juan de Plan deposit shows variations in $\mathrm{Ni}: \mathrm{Fe}$ ratio from 0 to 46.5 , whereas the gersdorffite compositions of Wagner \& Lorenz (2002) range between 0 and 44.0. These values indicate that both $\mathrm{Fe}$ and $\mathrm{Ni}$ are independent of each other, and that the constant $\mathrm{Ni}: \mathrm{Fe}$ ratio is not an invariant feature of the ternary sulfarsenides (Hem 1998), which is in contrast with those trends reported in the literature: Béziat et al. (1996) determined an almost fixed

\begin{tabular}{|c|c|c|c|c|c|c|c|c|c|c|c|c|c|}
\hline & & \multicolumn{6}{|c|}{ Compositions (weight \%) } & \multicolumn{5}{|c|}{ Atoms per formula unit } & \multirow[b]{2}{*}{ As\# } \\
\hline & & $\mathrm{S}$ & As & $\mathrm{Fe}$ & $\mathrm{Co}$ & $\mathrm{Ni}$ & Total & $\mathrm{S}$ & As & $\mathrm{Fe}$ & $\mathrm{Co}$ & $\mathrm{Ni}$ & \\
\hline $\begin{array}{l}\mathrm{GC}_{\mathrm{ss}} \mathrm{I} \\
n=89\end{array}$ & $\begin{array}{l}\min \\
\text { max } \\
\text { mean } \\
\text { C10 } \\
\text { C12 } \\
\text { C13 } \\
\text { C16 } \\
\text { C104 } \\
\text { C105 } \\
\text { C119 } \\
\text { C120 } \\
\text { C122 } \\
\text { C180 } \\
\text { C181 } \\
\text { C185 } \\
\text { D25 } \\
\text { D26 } \\
\text { D31 }\end{array}$ & $\begin{array}{l}15.38 \\
21.13 \\
18.71 \\
20.50 \\
20.20 \\
20.30 \\
21.10 \\
19.40 \\
16.20 \\
20.50 \\
20.10 \\
18.70 \\
20.00 \\
20.40 \\
20.00 \\
18.20 \\
18.10 \\
18.50\end{array}$ & $\begin{array}{l}44.19 \\
51.93 \\
46.59 \\
45.30 \\
44.95 \\
45.43 \\
44.19 \\
46.31 \\
49.48 \\
48.00 \\
45.36 \\
47.26 \\
44.58 \\
44.55 \\
45.25 \\
47.86 \\
46.91 \\
46.47\end{array}$ & $\begin{array}{r}1.21 \\
20.03 \\
8.26 \\
12.20 \\
11.70 \\
11.60 \\
12.60 \\
7.29 \\
1.21 \\
5.65 \\
12.80 \\
7.31 \\
12.70 \\
12.70 \\
12.40 \\
3.19 \\
3.43 \\
3.89\end{array}$ & $\begin{array}{r}3.73 \\
25.78 \\
11.75 \\
9.27 \\
6.55 \\
8.07 \\
5.84 \\
21.30 \\
16.00 \\
16.10 \\
9.73 \\
10.10 \\
6.44 \\
7.48 \\
8.01 \\
20.10 \\
23.00 \\
16.10\end{array}$ & $\begin{array}{r}2.22 \\
27.20 \\
14.50 \\
13.40 \\
17.00 \\
15.60 \\
16.80 \\
6.78 \\
13.40 \\
12.70 \\
12.70 \\
16.40 \\
15.50 \\
14.20 \\
14.50 \\
10.90 \\
7.57 \\
14.00\end{array}$ & $\begin{array}{r}97.02 \\
101.75 \\
100.09 \\
100.75 \\
100.64 \\
100.64 \\
100.94 \\
101.12 \\
99.53 \\
100.21 \\
100.89 \\
99.78 \\
99.63 \\
99.54 \\
100.23 \\
100.53 \\
99.41 \\
99.80\end{array}$ & $\begin{array}{l}0.83 \\
1.06 \\
0.97 \\
1.04 \\
1.03 \\
1.02 \\
1.06 \\
0.98 \\
1.01 \\
1.05 \\
1.02 \\
0.97 \\
1.02 \\
1.05 \\
1.02 \\
0.95 \\
0.96 \\
0.98\end{array}$ & $\begin{array}{l}0.95 \\
1.20 \\
1.04 \\
0.97 \\
0.98 \\
0.98 \\
0.95 \\
1.02 \\
1.10 \\
0.98 \\
0.98 \\
1.06 \\
0.99 \\
0.97 \\
0.98 \\
1.07 \\
1.06 \\
1.04\end{array}$ & $\begin{array}{l}0.03 \\
0.58 \\
0.25 \\
0.36 \\
0.34 \\
0.34 \\
0.37 \\
0.21 \\
0.03 \\
0.16 \\
0.37 \\
0.22 \\
0.38 \\
0.38 \\
0.36 \\
0.10 \\
0.10 \\
0.12\end{array}$ & $\begin{array}{l}0.10 \\
0.74 \\
0.33 \\
0.26 \\
0.18 \\
0.23 \\
0.16 \\
0.59 \\
0.46 \\
0.44 \\
0.27 \\
0.28 \\
0.18 \\
0.21 \\
0.23 \\
0.57 \\
0.66 \\
0.46\end{array}$ & $\begin{array}{l}0.07 \\
0.80 \\
0.41 \\
0.37 \\
0.47 \\
0.44 \\
0.47 \\
0.20 \\
0.39 \\
0.36 \\
0.35 \\
0.47 \\
0.43 \\
0.39 \\
0.41 \\
0.32 \\
0.22 \\
0.40\end{array}$ & $\begin{array}{l}0.47 \\
0.59 \\
0.52 \\
0.48 \\
0.49 \\
0.49 \\
0.47 \\
0.51 \\
0.52 \\
0.48 \\
0.49 \\
0.52 \\
0.49 \\
0.48 \\
0.49 \\
0.53 \\
0.52 \\
0.51\end{array}$ \\
\hline $\begin{array}{l}\mathrm{GC}_{\mathrm{ss}} \mathrm{II} \\
n=119\end{array}$ & $\begin{array}{l}\min \\
\text { max } \\
\text { mean } \\
\text { B36 } \\
\text { C9 } \\
\text { C11 } \\
\text { C15 } \\
\text { C148 } \\
\text { C149 } \\
\text { C152 } \\
\text { C184 } \\
\text { D57 } \\
\text { D61 } \\
\text { D63 } \\
\text { D105 } \\
\text { D106 } \\
\text { D107 } \\
\text { D108 } \\
\text { D109 } \\
\text { D110 } \\
\text { D169 }\end{array}$ & $\begin{array}{l}11.84 \\
19.53 \\
15.90 \\
14.40 \\
14.90 \\
12.32 \\
11.84 \\
18.36 \\
14.23 \\
15.60 \\
16.59 \\
13.68 \\
13.53 \\
14.90 \\
15.73 \\
14.69 \\
15.04 \\
16.29 \\
13.13 \\
14.85 \\
13.87\end{array}$ & $\begin{array}{l}45.39 \\
56.36 \\
50.51 \\
50.41 \\
52.49 \\
55.60 \\
56.36 \\
48.25 \\
53.00 \\
50.49 \\
49.32 \\
53.76 \\
54.62 \\
52.68 \\
51.09 \\
52.71 \\
52.30 \\
50.23 \\
54.51 \\
52.98 \\
53.62\end{array}$ & $\begin{array}{l}0.01 \\
9.60 \\
1.62 \\
3.74 \\
4.12 \\
1.36 \\
1.00 \\
7.50 \\
0.81 \\
1.10 \\
2.18 \\
0.58 \\
0.05 \\
1.82 \\
0.08 \\
0.15 \\
0.14 \\
0.04 \\
0.16 \\
0.02 \\
0.05\end{array}$ & $\begin{array}{r}0.58 \\
28.26 \\
10.72 \\
6.37 \\
7.05 \\
4.03 \\
5.44 \\
4.47 \\
3.53 \\
4.06 \\
4.06 \\
3.69 \\
3.16 \\
4.90 \\
1.26 \\
1.45 \\
1.41 \\
1.40 \\
1.55 \\
1.48 \\
6.76\end{array}$ & $\begin{array}{r}3.45 \\
32.73 \\
20.99 \\
23.10 \\
21.30 \\
26.06 \\
25.00 \\
21.20 \\
27.50 \\
26.70 \\
26.10 \\
28.90 \\
29.90 \\
26.50 \\
31.90 \\
32.20 \\
31.70 \\
32.20 \\
31.50 \\
31.23 \\
26.41\end{array}$ & $\begin{array}{r}98.10 \\
101.47 \\
99.95 \\
98.19 \\
100.20 \\
99.69 \\
100.20 \\
99.94 \\
99.12 \\
98.10 \\
98.46 \\
100.82 \\
101.37 \\
100.86 \\
100.78 \\
101.29 \\
100.84 \\
100.42 \\
101.00 \\
100.56 \\
100.73\end{array}$ & $\begin{array}{l}0.67 \\
1.01 \\
0.85 \\
0.80 \\
0.81 \\
0.69 \\
0.67 \\
0.96 \\
0.78 \\
0.86 \\
0.90 \\
0.75 \\
0.74 \\
1.08 \\
0.85 \\
0.80 \\
0.82 \\
0.87 \\
0.72 \\
0.80 \\
0.75\end{array}$ & $\begin{array}{l}1.01 \\
1.36 \\
1.17 \\
1.19 \\
1.23 \\
1.35 \\
1.36 \\
1.08 \\
1.26 \\
1.18 \\
1.14 \\
1.26 \\
1.28 \\
1.22 \\
1.18 \\
1.21 \\
1.21 \\
1.15 \\
1.28 \\
1.23 \\
1.26\end{array}$ & $\begin{array}{l}0.00 \\
0.28 \\
0.05 \\
0.12 \\
0.12 \\
0.04 \\
0.04 \\
0.22 \\
0.02 \\
0.04 \\
0.07 \\
0.02 \\
0.00 \\
0.05 \\
0.00 \\
0.00 \\
0.00 \\
0.00 \\
0.00 \\
0.00 \\
0.00\end{array}$ & $\begin{array}{l}0.02 \\
0.81 \\
0.31 \\
0.20 \\
0.21 \\
0.13 \\
0.16 \\
0.13 \\
0.11 \\
0.12 \\
0.12 \\
0.11 \\
0.09 \\
0.14 \\
0.03 \\
0.03 \\
0.03 \\
0.03 \\
0.05 \\
0.05 \\
0.19\end{array}$ & $\begin{array}{l}0.10 \\
0.96 \\
0.62 \\
0.69 \\
0.63 \\
0.80 \\
0.78 \\
1.61 \\
0.83 \\
0.79 \\
0.76 \\
0.86 \\
0.89 \\
0.78 \\
0.94 \\
0.95 \\
0.94 \\
0.94 \\
0.95 \\
0.93 \\
0.79\end{array}$ & $\begin{array}{l}0.50 \\
0.67 \\
0.58 \\
0.60 \\
0.60 \\
0.66 \\
0.67 \\
0.53 \\
0.62 \\
0.58 \\
0.56 \\
0.63 \\
0.63 \\
0.60 \\
0.58 \\
0.60 \\
0.60 \\
0.57 \\
0.64 \\
0.61 \\
0.63\end{array}$ \\
\hline
\end{tabular}

Representative results of micro-analyses have been indicated in Figures $2 \mathrm{~A}, 2 \mathrm{C}, 2 \mathrm{E}, 2 \mathrm{~F}, 2 \mathrm{G}, 2 \mathrm{H}, 2 \mathrm{I}$, and 2J. As\#: $\mathrm{As} /(\mathrm{As}+\mathrm{S})$. 

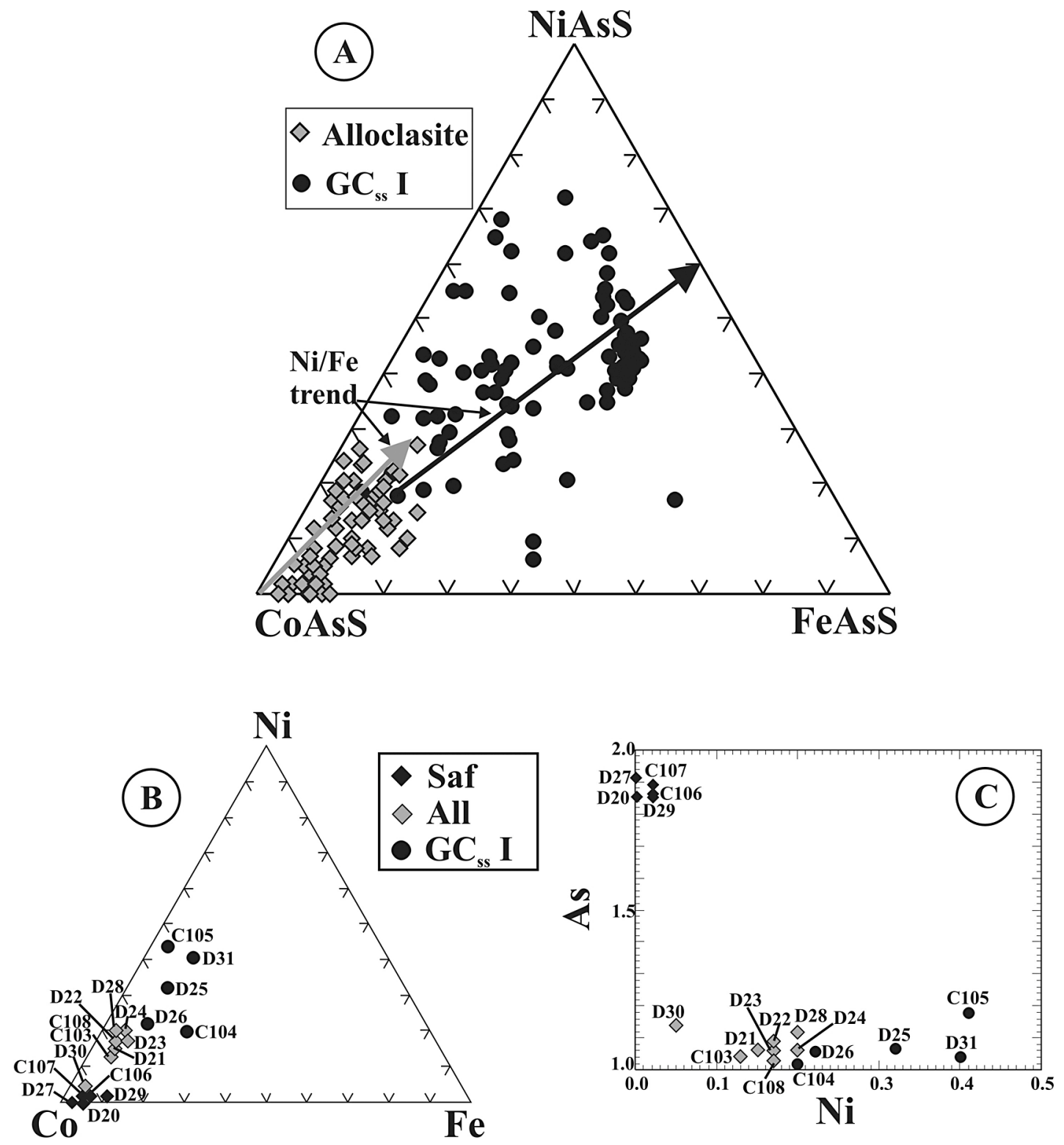

FIG. 5. A. Plot of the composition of alloclasite and $\mathrm{GC}_{\mathrm{ss}} \mathrm{I}$ crystals in the system NiAsS-CoAsS-FeAsS (in atomic proportions). The arrows represent the trends that indicate the compositional variability in both minerals. B. Composition of safflorite, alloclasite and $\mathrm{GC}_{\mathrm{ss}} \mathrm{I}$ in atomic proportions. The numbers represent the compositions listed in Tables 3, 4 and 5 and indicated in Figure 2E. C. Binary diagram showing the relationship between As and Ni in atoms per unit formula.

$\left(\mathrm{Fe}_{0.33} \mathrm{Ni}_{0.67}\right) \leftrightarrow$ Co trend for solid solutions of cobaltite-gersdorffite with a Co content of 0.05 to 0.59 , and corresponding trends are shown by the data of Mposkos (1983) and Gervilla et al. (1996). Fukuoka \& Hirowatari (1980) found that the $\mathrm{Fe}_{0.35} \mathrm{Ni}_{0.65}$ line defines the maximum Ni:Fe ratio. Béziat et al. (1996) argued that it was caused by the substitution of $3 \mathrm{Co}$ atoms by $2 \mathrm{Ni}$ atoms and $1 \mathrm{Fe}$ atom.

With regard to As:S ratio, the San Juan de Plan gersdorffite-cobaltite $\mathrm{ss}$ shows narrower ranges $\left(\mathrm{GC}_{\mathrm{ss}} \mathrm{I}\right.$ : 0.9 to $1.45, \mathrm{GC}_{\mathrm{ss}}$ II: 1.00 to 2.03$)$ than those values $(0.78$ to 2.44$)$ reported previously by Barkov et al. (1999, and 

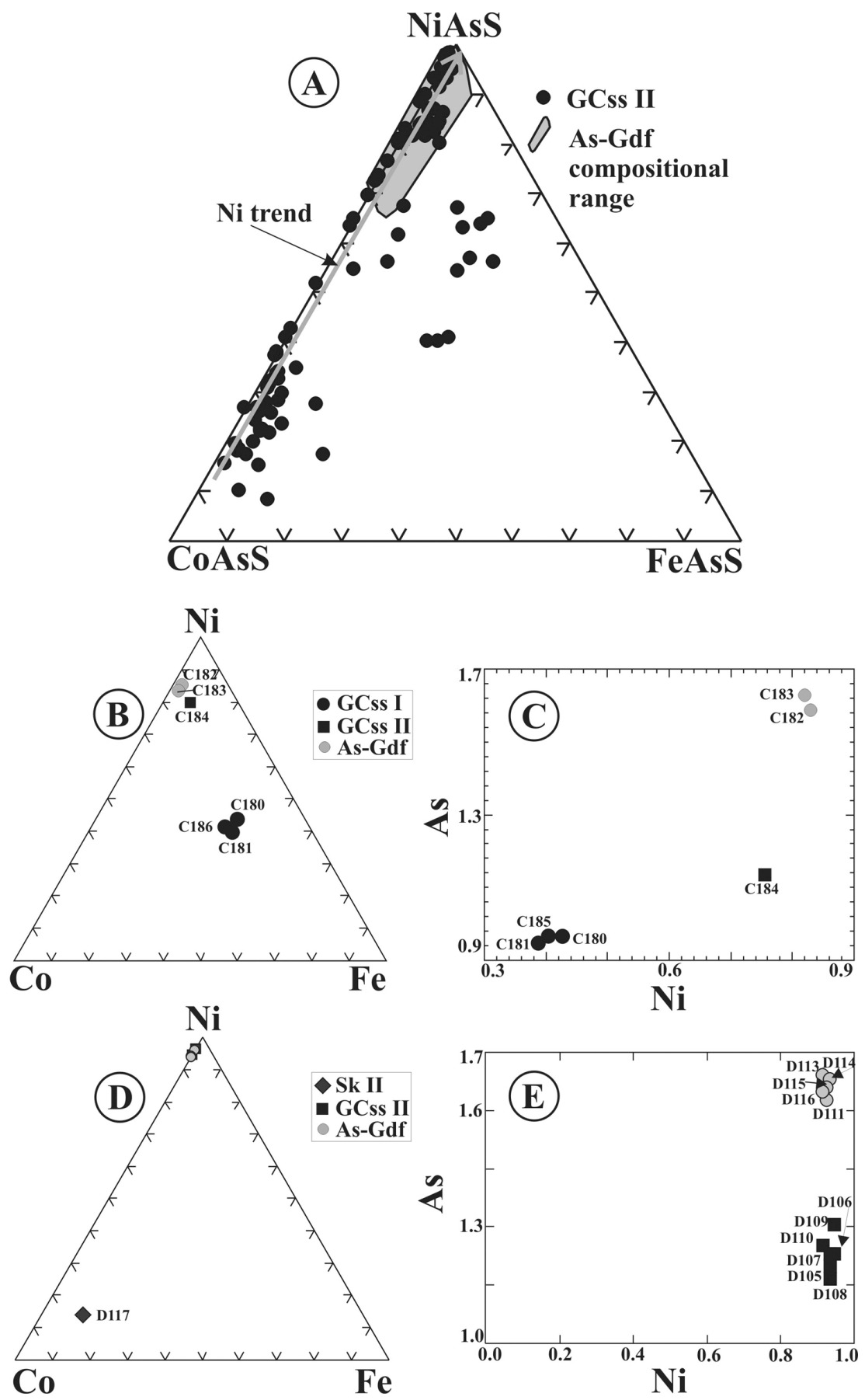

FIG. 6. A. Composition of $\mathrm{GC}_{\mathrm{ss}} \mathrm{II}$ and As-Gdf crystals in the system NiAsS-CoAsS-FeAsS. The arrow represents the Ni trend. B. Composition of $\mathrm{GC}_{\mathrm{ss}} \mathrm{I}, \mathrm{GC}_{\mathrm{ss}} \mathrm{II}$ and As-Gdf in atomic proportions. The numbers refer to compositions listed in Tables 5 and 6 and indicated in Figure 2H. C. Binary diagram showing the relationship between As and $\mathrm{Ni}$ in atomic proportions. D. Composition of $\mathrm{GC}_{\mathrm{ss}}$ II, As-Gdf and Sk II (atomic proportions) for the case of figure 2G. E. Binary diagram showing the compositional variation in As and Ni contents (in atoms per unit formula) between GCss II and As-Gdf crystals, from Figure 2G. 
TABLE 6. STATISTICAL RESULTS OF ELECTRON-MICROPROBE ANALYSES OF ARSENIC-RICH GERSDORFFITE (As-Gdf, STAGE IV) FROM THE SAN JUAN DE PLAN DEPOSIT, CENTRAL PYRENEES, SPAIN

\begin{tabular}{|c|c|c|c|c|c|c|c|c|c|c|c|c|}
\hline & \multicolumn{6}{|c|}{ Compositions (weight \%) } & \multicolumn{5}{|c|}{ Atoms per formula unit } & \multirow[b]{2}{*}{ As\# } \\
\hline & $\mathrm{S}$ & As & $\mathrm{Fe}$ & Co & $\mathrm{Ni}$ & Total & $\mathrm{S}$ & As & $\mathrm{Fe}$ & $\mathrm{Co}$ & $\mathrm{Ni}$ & \\
\hline $\begin{array}{ll}\text { As-Gdf } \\
n=157 & \min \\
& \text { mean } \\
\text { B } 40 \\
\text { C121 } \\
\text { C150 } \\
\text { C151 } \\
\text { C154 } \\
\text { C182 } \\
\text { C183 } \\
\text { D58 } \\
\text { D60 } \\
\text { D62 } \\
\text { D111 } \\
\text { D113 } \\
\text { D114 } \\
\text { D115 } \\
\text { D116 } \\
\text { D170 } \\
\text { D171 }\end{array}$ & $\begin{array}{r}4.43 \\
13.85 \\
8.33 \\
6.53 \\
11.92 \\
5.78 \\
7.05 \\
5.24 \\
7.50 \\
6.52 \\
6.03 \\
5.97 \\
5.74 \\
7.46 \\
6.26 \\
6.46 \\
6.64 \\
7.17 \\
6.37 \\
7.52\end{array}$ & $\begin{array}{l}53.44 \\
66.29 \\
60.81 \\
62.63 \\
56.04 \\
64.54 \\
62.43 \\
64.93 \\
61.94 \\
63.24 \\
64.58 \\
64.43 \\
65.17 \\
62.49 \\
64.08 \\
63.53 \\
63.73 \\
62.54 \\
63.59 \\
62.25\end{array}$ & $\begin{array}{l}0.00 \\
1.66 \\
0.27 \\
0.32 \\
0.84 \\
0.06 \\
0.08 \\
0.00 \\
0.47 \\
0.47 \\
0.07 \\
0.05 \\
0.03 \\
0.00 \\
0.08 \\
0.05 \\
0.06 \\
0.13 \\
0.04 \\
0.08\end{array}$ & $\begin{array}{l}0.53 \\
8.61 \\
3.21 \\
2.43 \\
6.92 \\
2.60 \\
2.48 \\
2.20 \\
3.26 \\
3.85 \\
2.51 \\
2.68 \\
2.48 \\
1.80 \\
1.56 \\
1.44 \\
1.49 \\
0.56 \\
1.89 \\
1.83\end{array}$ & $\begin{array}{l}20.08 \\
30.88 \\
26.76 \\
27.14 \\
22.83 \\
25.58 \\
26.20 \\
26.01 \\
25.06 \\
24.67 \\
27.76 \\
28.04 \\
27.78 \\
28.79 \\
28.37 \\
28.73 \\
28.73 \\
28.29 \\
28.63 \\
28.91\end{array}$ & $\begin{array}{r}98.12 \\
101.38 \\
99.74 \\
99.31 \\
98.70 \\
98.65 \\
98.56 \\
98.4 \\
98.41 \\
99.07 \\
101.05 \\
101.20 \\
101.38 \\
100.68 \\
100.52 \\
100.27 \\
100.90 \\
99.89 \\
100.42 \\
100.84\end{array}$ & $\begin{array}{l}0.28 \\
0.77 \\
0.49 \\
0.39 \\
0.67 \\
0.36 \\
0.43 \\
0.32 \\
0.44 \\
0.39 \\
0.37 \\
0.36 \\
0.35 \\
0.44 \\
0.38 \\
0.38 \\
0.40 \\
0.42 \\
0.38 \\
0.44\end{array}$ & $\begin{array}{l}1.28 \\
1.75 \\
1.54 \\
1.63 \\
1.36 \\
1.70 \\
1.62 \\
1.73 \\
1.60 \\
1.64 \\
1.65 \\
1.63 \\
1.67 \\
1.58 \\
1.64 \\
1.63 \\
1.61 \\
1.60 \\
1.62 \\
1.58\end{array}$ & $\begin{array}{l}0.00 \\
0.06 \\
0.01 \\
0.02 \\
0.04 \\
0.00 \\
0.00 \\
0.00 \\
0.00 \\
0.00 \\
0.00 \\
0.00 \\
0.00 \\
0.00 \\
0.00 \\
0.00 \\
0.00 \\
0.00 \\
0.00 \\
0.00\end{array}$ & $\begin{array}{l}0.02 \\
0.27 \\
0.10 \\
0.08 \\
0.22 \\
0.08 \\
0.08 \\
0.08 \\
0.12 \\
0.14 \\
0.08 \\
0.09 \\
0.08 \\
0.06 \\
0.06 \\
0.04 \\
0.06 \\
0.06 \\
0.06 \\
0.06\end{array}$ & $\begin{array}{l}0.65 \\
0.98 \\
0.86 \\
0.89 \\
0.71 \\
0.87 \\
0.88 \\
0.87 \\
0.83 \\
0.82 \\
0.90 \\
0.91 \\
0.90 \\
0.93 \\
0.92 \\
0.94 \\
0.93 \\
0.92 \\
0.94 \\
0.93\end{array}$ & $\begin{array}{l}0.62 \\
0.89 \\
0.78 \\
0.81 \\
0.67 \\
0.83 \\
0.79 \\
0.84 \\
0.78 \\
0.81 \\
0.82 \\
0.82 \\
0.83 \\
0.78 \\
0.81 \\
0.81 \\
0.80 \\
0.79 \\
0.81 \\
0.78\end{array}$ \\
\hline
\end{tabular}

Representative results of micro-analyses are indicated in Figures $2 \mathrm{C}, 2 \mathrm{~F}, 2 \mathrm{G}, 2 \mathrm{H}$, and $2 \mathrm{~J}$. As\#: As/(As + S).

references therein) and Béziat et al. (1996, and references therein). However, the As:S ratio of $\mathrm{GC}_{\mathrm{ss}}$ II is similar to that of Wagner \& Lorenz (2002), which varies from 1.23 to 1.76 . The experimental results of Maurel \& Picot (1974), and the data reported by Béziat et al. (1996) and Barkov et al. (1999), show that the degree of substitution of As for $\mathrm{S}$ increases with increasing $(\mathrm{Ni}+\mathrm{Fe})$ content, which contrasts with our results.

In order to know the scheme of substitution in the $\mathrm{GC}_{\mathrm{ss}}$ crystals, the limits of substitution, and the compositional trends related to such substitutions, we have plotted $M e\left(\mathrm{Ni}\right.$ or Co contents versus $\mathrm{As}_{\text {total }}-\mathrm{As}_{\text {MeAsS }}$ (Figs. 7A, B). Thus, the covariation between $M e$ and As contents, as well as the deviation from $[\mathrm{AsS}]^{3-}$ toward either $\left[\mathrm{As}_{2}\right]^{4-}$ or $\left[\mathrm{S}_{2}\right]^{2-}$ anionic pairs, is revealed. Thin dotted lines in Figures 7A and 7B represent $\mathrm{MeAs}_{2}$, $\mathrm{MeAsS}$ and $\mathrm{MeS}_{2}$ binary systems in which anionic content is stoichiometric, i.e., only cationic substitution is allowed. Thick dashed and solid lines show regression lines for $\mathrm{GC}_{\mathrm{ss}}$ I and $\mathrm{GC}_{\mathrm{ss}}$ II, respectively. Since regression lines are not parallel to stoichiometric lines, cations and anions are mutually dependent and, therefore, a coupled substitution $\left(\mathrm{MeAsS} \leftrightarrow \mathrm{MeAs}_{2}\right.$ or $\mathrm{MeAsS} \leftrightarrow$ $\mathrm{MeS}_{2}$ ) is deduced. The slope of regression lines provides information about the proportion of each pattern of substitution; thus, in Figure 7A, the value 1.25929 for $\mathrm{GC}_{\mathrm{ss}}$ II indicates that the contribution of coupled substitution $\left[(\mathrm{Co}, \mathrm{Fe}) \mathrm{AsS} \leftrightarrow \mathrm{NiAs}_{2}\right]$ is $c a .25 \%$ of the total. In the case of $\mathrm{GC}_{\mathrm{ss}} \mathrm{I}$, the contribution of the coupled substitution is smaller. In Figure 7B, the ordinate gives the As content related to Co content after considering the As contribution linked to Ni content (see Fig. 7A), and to stoichiometric FeAsS. The relationship between regres- sion and stoichiometric lines in $\mathrm{GC}_{\mathrm{ss}} \mathrm{I}$ crystals indicates that the Co-free end-member has an As deficit of $c a$. 0.1 apfu, i.e., there is CoAsS $\leftrightarrow \mathrm{FeS}_{2}$ substitution. However, from the crossover point between the above lines (0.6 Co $a p f u$ ) upward, there exists a surplus of As. In addition, the slope of 1.17003 for the Co-end member indicates that the extent of substitution $\mathrm{CoAsS} \leftrightarrow \mathrm{CoAs}_{2}$ is $c a .7 \%$. Consequently, it could be deduced that the increase in $\left[\mathrm{S}_{2}\right]^{2-}$ is linked to the introduction of Fe into the $\mathrm{GC}_{\mathrm{ss}}$ I structure. With regard to the $\mathrm{GC}_{\mathrm{ss}}$ II data, the slope (1.0262) of the regression line that runs parallel to the CoAsS stoichiometric line indicates that cationic substitution is the only scheme of substitution.

To sum up, the scheme of substitution of $\mathrm{GC}_{\mathrm{ss}} \mathrm{I}$ and $\mathrm{GC}_{\mathrm{ss}}$ II crystals is slightly different; it indicates a positive correlation between $\mathrm{S}$ and $\mathrm{Fe}$ contents, and $\mathrm{Co}$ and As contents, in $\mathrm{GC}_{\mathrm{ss}}$ I crystals, whereas $\mathrm{GC}_{\mathrm{ss}}$ II crystals show a positive correlation between $\mathrm{As}$ and $\mathrm{Ni}$ contents. From Figures 7A and 7B, it follows that $\mathrm{GC}_{\mathrm{ss}} \mathrm{II}$ is more As-rich than $\mathrm{GC}_{\mathrm{ss}} \mathrm{I}$, and that the highest As:S ratio corresponds to $\mathrm{Fe}$-free $\mathrm{GC}_{\mathrm{ss}}$ II. Consequently, following Hem et al. (2001), we believe that these variations are related to the As fugacity in the mineralizing fluids. An increase in the As fugacity during deposition of $\mathrm{GC}_{\mathrm{ss}}$ II favored the incorporation of $\mathrm{Ni}$ in $\mathrm{GC}_{\mathrm{ss}}$ II instead of $\mathrm{Co}$ (Ni trend). Thus, a higher fugacity of As in the fluid results in a higher content of As and $\mathrm{Ni}$ in the crystallizing mineral. This enrichment resulted in the subsequent crystallization of As-rich gersdorffite and rammelsbergite during stage IV.

Another discrepancy among our data and the experimental data reported in literature concerns the compositional variability of sulfarsenides and diarsenides. Yund 
(1962) determined that the gersdorffite solid-solution extends along a line from rammelsbergite $\left(\mathrm{NiAs}_{2}\right)$ to vaesite $\left(\mathrm{NiS}_{2}\right)$ in the system $\mathrm{Ni}-\mathrm{As}-\mathrm{S}$. Likewise, Yund (1962) determined experimentally that the maximum As content of gersdorffite corresponds to the formula $\mathrm{NiAs}_{1.77} \mathrm{~S}_{0.23}(66.74 \mathrm{wt} \%$ As and $3.71 \mathrm{wt} \% \mathrm{~S}$ ), whereas the maximum $\mathrm{S}$ content of rammelsbergite is $1.1 \pm 0.1$ $\mathrm{wt} \%$ at $700^{\circ} \mathrm{C}\left(\mathrm{As}_{1.93} \mathrm{~S}_{0.07}\right)$. Consequently, Yund (1962) proposed a miscibility gap between $\mathrm{As}_{1.93} \mathrm{~S}_{0.07}$ and $\mathrm{As}_{1.77} \mathrm{~S}_{0.23}$. However, Spiridonov \& Chvileva (1995) found a complete solid-solution between gersdorffite and krutovite (cubic $\mathrm{NiAs}_{2}$ ) in samples from the Manybay U-Mo deposit, in Kazakhstan. Recently, Hem \& Makovicky (2004) found experimentally a nickel diarsenide containing 12-14 at.\% S marked by optical similarity with gersdorffite, which confirmed the existence of krutovite (cubic NiAs 2 phase, $\mathrm{Pa3}$ ). Electronmicroprobe analyses (Figs. 7C, D) seemingly show that there exists a compositional continuum between rammelsbergite and gersdorffite. However, microscopic observations (complex twinning) suggest to us that rammelsbergite is a product of inversion of krutovite. Consequently, our study supports the findings of Spiridonov \& Chvileva (1995) on the complete solidsolution between gersdorffite and krutovite.

With regard to the compositional variability of gersdorffite, Choi \& Imai (1985) reported an arsenic content of $63 \mathrm{wt} \%\left(\mathrm{As}_{1.61} \mathrm{~S}_{0.37}\right)$. Arsenic contents of gersdorffite in the literature are invariably lower than those of gersdorffite from San Juan de Plan $(66.29 \mathrm{wt} \%)$, which, in turn, is as high as that experimentally proposed by Yund (1962). Consequently, the As:S ratio in our gersdorffite spans a range from 2.14 to 6.25 , which is broader than that found in the literature [Bayliss (1982): 0.97 to 2.44 , Petruk et al. (1971): 1.15 to 4.0 , Choi \& Imai (1985): 1.02 to 4.35 , Vinogradova et al. (1974):
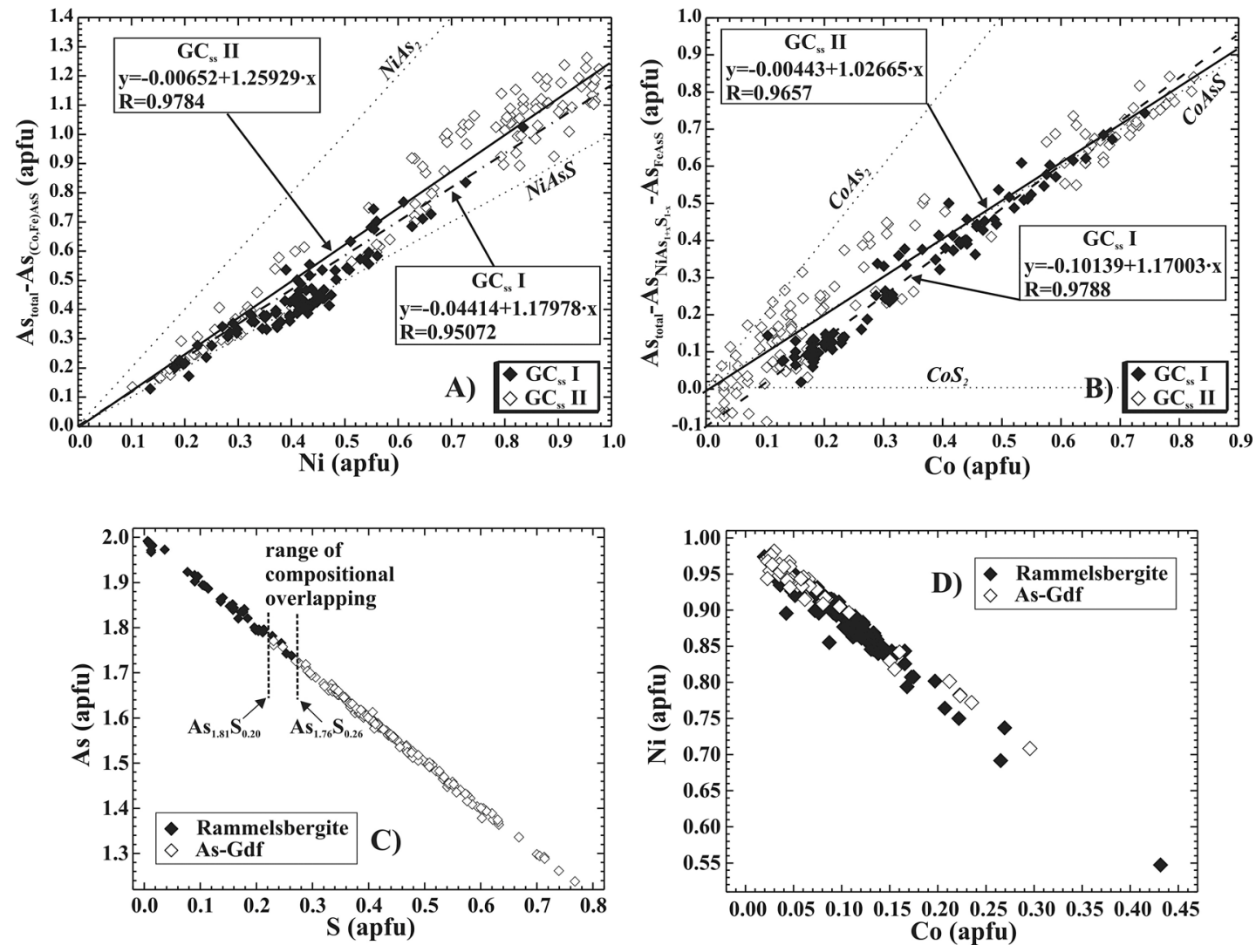

FIG. 7. A. Plot of $\mathrm{As}_{\mathrm{total}}-\mathrm{As}_{(\mathrm{Co}, \mathrm{Fe}) \mathrm{Ass}}$ versus $\mathrm{Ni}$, in atoms per formula unit, for $\mathrm{GC}_{\mathrm{ss}}$ crystals. Regression lines with their $\mathrm{R}$ values along with the $M e \mathrm{As}_{2}, \mathrm{Me} \mathrm{AsS}$ and $M e \mathrm{~S}_{2}$ binary systems have also been represented. B. Plot of $\mathrm{As}_{\mathrm{total}}-\mathrm{As}_{\mathrm{NiAs} 1+x} \mathrm{~S}_{1-x}-\mathrm{As}_{\mathrm{FeAsS}}$ versus $\mathrm{Co}$, in atoms per formula unit. C. Plot of As versus $\mathrm{S}$ (in atoms per formula unit) for rammelsbergite (solid diamond) and arsenic-rich gersdorffite (open diamond). D. Binary diagram showing the relationship between $\mathrm{Ni}$ and Co in atoms per formula unit for the aforementioned minerals. 
1.5 to 2.4 , Wagner \& Lorenz (2002): 1.26 to 1.77 , Oen et al. (1971), Ixer et al. (1979), Permingeat \& Zehni (1988), Gervilla \& Rønsbo (1992), Béziat et al. (1996), Barkov et al. (1999): <1.44]. Figure 8 shows that sulfarsenides have low Ni contents, whereas the value $A s: S$ is close to 1 . However, as this ratio increases, so does the Ni content, displaying a continuous variation of the As:S ratio from $\mathrm{GC}_{\mathrm{ss}}$ II to As-rich gersdorffite and rammelsbergite. Data in Figure 8 also suggest that As-Gdf represents the As-rich limit of $\mathrm{GC}_{\mathrm{ss}}$ II at $650^{\circ} \mathrm{C}$, according to the experimental results of Hem \& Makovicky (2004). These results further support the conclusion that both As and Ni contents in gersdorffitecobaltite $_{\text {ss }}$ are controlled by the As fugacity of the mineralizing fluid at high temperature.

Figure 9 shows the binary presentation of the sides of the $M X_{2}$ prism at $650^{\circ} \mathrm{C}$ from Hem \& Makovicky (2004), with the solid lines showing the limits in solid solution for a given association of phases. The compositions of rammelsbergite, As-Gdf, $\mathrm{GC}_{\mathrm{ss}}$ I and $\mathrm{GC}_{\mathrm{ss}}$ II from San Juan de Plan deposit have been plotted in this diagram. $\mathrm{GC}_{\mathrm{ss}} \mathrm{I}$ and $\mathrm{GC}_{\mathrm{ss}}$ II display a solid solution that fills the gersdorffite-cobaltite field obtained by Hem \& Makovicky (2004), though, as we have noted, the As contents are higher than in our samples. In addition, AsGdf and rammelsbergite compositions not only overlap the solid-solution field between gersdorffite and krutovite-rammelsbergite, but also extend toward compositions richer in As and Co plus Fe than those investigated by Hem \& Makovicky (2004). This fact also supports the hypothesis that the presence of rammelsbergite instead of krutovite in San Juan de Plan ores may be due to the inversion of krutovite to rammelsbergite.

In the San Juan de Plan ores, the assemblage crystallized during stage IV resembles that described by Hem $\&$ Makovicky (2004) at $650^{\circ} \mathrm{C}$ (Fig. 9), since smallscale bands of As-rich gersdorffite containing up to 51.94 at.\% As alternate in zoned crystals with another Ni diarsenide (Fig. 2G). This finding may explain the larger extension of our As-Gdf data toward the compositions rich in As for the homogeneous phases plotted in Figure $2 \mathrm{G}$.

\section{Conditions of ore formation}

The paragenetic sequence at San Juan de Plan is shown with the variation in arsenic fugacity during the precipitation of the different paragenetic stages in Figure 1. Keeping in mind that the arsenopyrite studied fulfills the criteria of Kretschmar \& Scott (1976) and Sharp et al. (1985), that the minor element content is

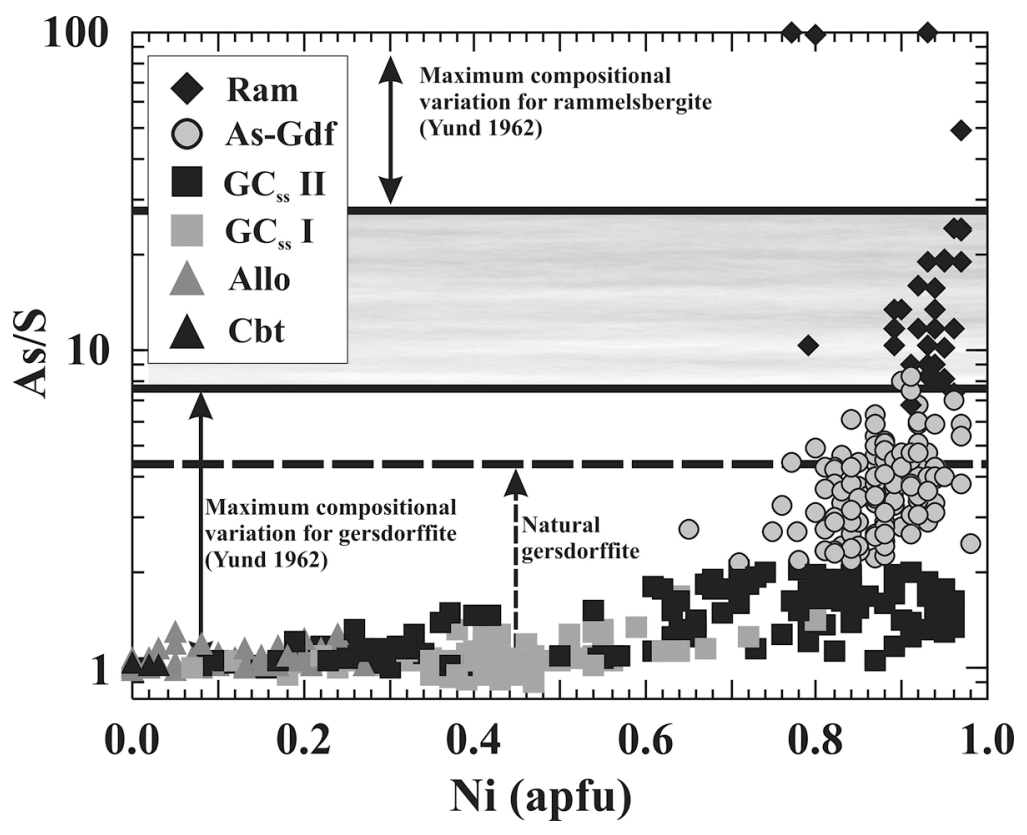

FIG. 8. As/S relationship versus $\mathrm{Ni}$ (in atoms per unit formula) in a linear-log scale. Solid lines represent empirical compositional limits for rammelsbergite and gersdorffite (Yund 1962). The dashed line shows the maximum compositional variation in gersdorffite found in the literature. The shaded area represents the hypothetical miscibility-gap between rammelsbergite and gersdorffite. 
less than $1 \mathrm{wt} \%$ and there is no textural zoning, the extent of As-for-S substitution has been used as a geothermometer. It yields a range of temperatures of formation between 325 and $425^{\circ} \mathrm{C}$ for stage I. At this temperature, $\log f\left(\mathrm{~S}_{2}\right)$ ranges between -9.2 and -6.2 for the given phase-assemblage.

The crystallization of nickeline, skutterudite I and safflorite during stage II provide strong support for a decrease in $f\left(S_{2}\right)$ and, therefore, a strong increase in $f$ (As). According to Hem \& Makovicky (2004), Co-rich safflorite at $650^{\circ} \mathrm{C}$ exhibits a maximum sulfur content of 0.18 apfu (5.92 at.\%), whereas at $500^{\circ} \mathrm{C}$ it can contain up to 5.1 at. $\% \mathrm{~S}$. The maximum sulfur content of safflorite from San Juan de Plan, 0.16 apfu (5.37 at.\%), suggests formation temperatures close to $550^{\circ} \mathrm{C}$.

According to the experiments of Maurel \& Picot (1974) and Hem \& Makovicky (2004), the maximum temperature of ore formation should have been reached when alloclasite was deposited during stage III and in stage IV during the crystallization of As-rich gersdorffite coexisting with rammelsbergite. The reported temperatures of alloclasite crystallization vary from $300-400^{\circ} \mathrm{C}$ (Ixer et al. 1979 ) to $450-600^{\circ} \mathrm{C}$ (Petruk et al. 1971). Nevertheless, Maurel \& Picot (1974) found alloclasite to be a high-temperature phase, coexisting with cobaltite or safflorite at temperatures above $800^{\circ} \mathrm{C}$. They also found that alloclasite is stable only on the As-rich side of the $\mathrm{CoS}_{2}-\mathrm{CoAs}_{2}$ binary join and, consequently, an excess of As is necessary for the formation of this mineral; on the other hand, high $\mathrm{Ni}$ contents inhibit the formation of alloclasite owing to the neutralization of As by $\mathrm{Ni}$ where the ratio 1:1 between them is reached. Our data partially agree with the results of Maurel \& Picot (1974), since alloclasite in the
San Juan de Plan deposit are enriched in As and displays low Ni contents. Hem \& Makovicky (2004) found that the As content of alloclasite, at $650^{\circ} \mathrm{C}$, varies from 37 to 54 at.\%, and between 42 and 52 at. $\%$ at $500^{\circ} \mathrm{C}$ (our compositions range between 33 and 38 at.\%). However, Hem \& Makovicky (2004) recognized that their alloclasite is more enriched in As than that found in nature. The high As content of the alloclasite analyzed by Hem \& Makovicky (2004) with respect to that in the San Juan de Plan ores could indicate that the latter represent a S-rich extension of the alloclasite solid-solution field. Regarding Fe, Co, and Ni, Hem \& Makovicky (2004) found that at $650^{\circ} \mathrm{C}, \mathrm{Co}$ is completely replaced by a mixture of $\mathrm{Fe}$ and $\mathrm{Ni}$, whereas at $500^{\circ} \mathrm{C}$, both elements show a limited solubility $(\mathrm{Fe}<1.4$ at. $\%, \mathrm{Ni}<2.8$ at.\%). In our samples, $\mathrm{Fe}$ is less than 13.0 at.\% and $\mathrm{Ni}$, less than 8.8 at.\%, which equates to a temperature formation of alloclasite at San Juan de Plan between $650^{\circ}$ and $550^{\circ} \mathrm{C}$. This estimate must be treated with caution because, as noted above, alloclasite from San Juan de Plan ores crystallized in an environment richer in $\mathrm{S}$ that that studied by Hem \& Makovicky (2004). However, the value of the $c$ parameter of graphite (between 6.7157 and $6.7211 \AA$ ) from stylolitic graphite seams linked to the ore horizon has been used as a geothermometer (Shengelia et al. 1977). Calculated temperatures range between $650^{\circ}$ and $550^{\circ} \mathrm{C}$.

The compositions of $\mathrm{GC}_{\mathrm{ss}}$ I plot within the $650^{\circ} \mathrm{C}$ immiscibility region (Fig. 10A) in the system NiAsSCoAsS-FeAsS (Klemm 1965b). Misra \& Fleet (1975), Oen et al. (1984), and Hem et al. (2001) obtained contradictory temperatures when they applied Klemm's geothermometer. Misra \& Fleet (1975) suggested that Klemm (1965b) did not take into account the As-for-S

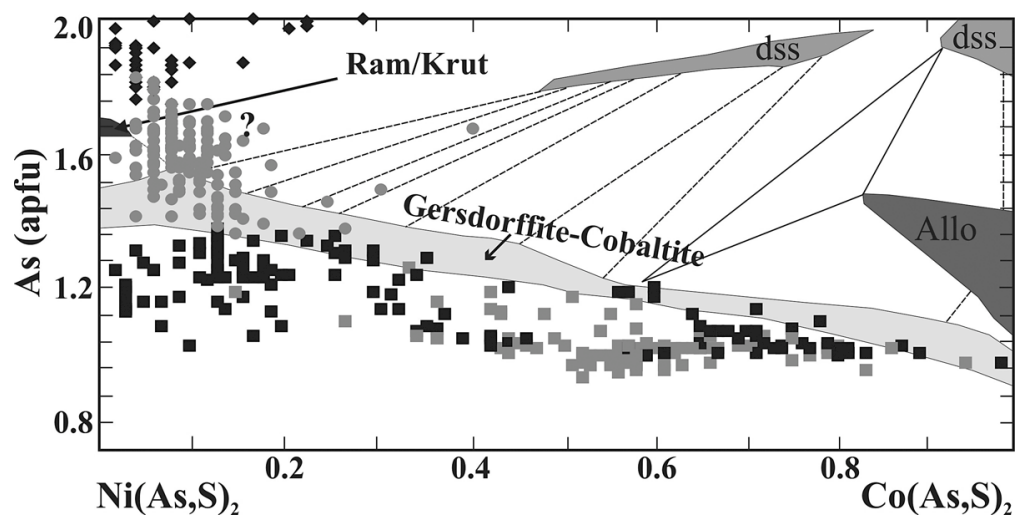

FIG. 9. The concentration of As (atoms per formula unit) versus compositional variations in terms of $\mathrm{Ni}-(\mathrm{Co}+\mathrm{Fe})$ for rammelsbergite (black diamond), As-Gdf (grey circle), $\mathrm{GC}_{\mathrm{ss}}$ $\mathrm{I}$ (grey square) and $\mathrm{GC}_{\mathrm{ss}} \mathrm{II}$ (black square). Diarsenides formed at $650^{\circ} \mathrm{C}$ in equilibrium with sulfarsenides and their solid-solution fields from Hem \& Makovicky (2004) have also been represented. 


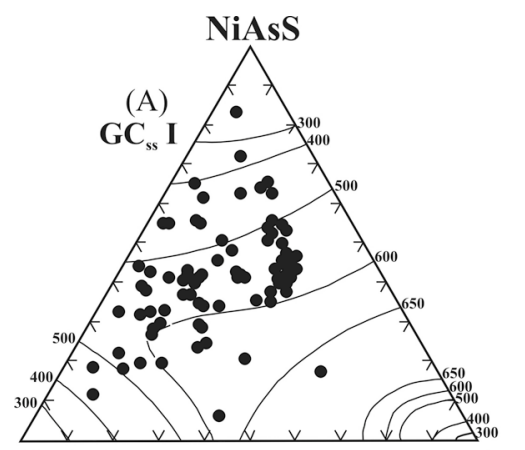

CoAsS

FeAsS

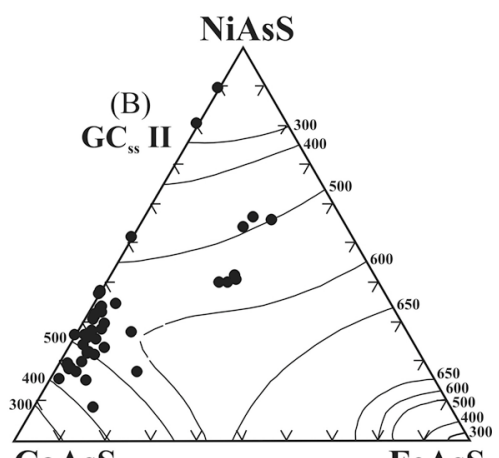

CoAsS

FeAsS

FIG. 10. Compositional plot of $\mathrm{GC}_{\mathrm{ss}} \mathrm{I}(\mathrm{A})$ and $\mathrm{GC}_{\mathrm{ss}} \mathrm{II}(\mathrm{B})$ in the system NiAsS-CoAsSFeAsS. Solvus lines at different temperatures are taken from Klemm (1965b).

substitution, which adds a degree of variance to the system, making his solvus diagram useless for geothermometry. Hem \& Makovicky (2004) found a nearly complete solid-solution between gersdorffite and cobaltite, again at $650^{\circ} \mathrm{C}$, with a linear correlation between $\mathrm{As}$ and $\mathrm{Ni}$ and a negative correlation between $\mathrm{Fe}$ and As. At $500^{\circ} \mathrm{C}$, the solid solution is very limited, and gersdorffite can contain up to 2.8 at.\% Fe and 5.4 at.\% $\mathrm{Co}$, whereas the As content is very high with respect to natural samples. As can be seen in Figure $5 \mathrm{~A}$, the $\mathrm{GC}_{\mathrm{ss}}$ I compositions show intermediate compositions between gersdorffite and cobaltite end-members, having $\mathrm{Fe}$ and Ni contents up to 19 and 26 at.\%, respectively, and with a negative correlation between As and Fe. These data suggest that $\mathrm{GC}_{\mathrm{ss}} \mathrm{I}$ formed after alloclasite under similar conditions of temperature.

During the formation of the stage-IV ore assemblage $\left(\mathrm{GC}_{\mathrm{ss}} \mathrm{II}\right.$, As-Gdf and rammelsbergite), the activity of arsenic increased without variations in the temperature. Although the rammelsbergite-pararammelsbergite inversion occurs at $590^{\circ} \mathrm{C}$ for pure $\mathrm{NiAs}_{2}$, the substitution of approximately $1 \mathrm{wt} \% \mathrm{~S}$ for As lowers the inversion temperature to $475 \pm 25^{\circ} \mathrm{C}$ (Yund 1962). As both Co and $\mathrm{Fe}$ also probably lower the inversion temperature, taking into account the compositions of San Juan de Plan rammelsbergite, the temperature of crystallization in the present assemblage must have remained close to 500$600^{\circ} \mathrm{C}$. When Klemm (1965b) investigated the temperature dependence of the (Fe,Co,Ni)AsS solid-solutions and suggested their application as a geothermometer, he did not deal with the As-for-S substitution. Consequently, if we try to apply his results, we must first leave out all those $\mathrm{GC}_{\mathrm{ss}} \mathrm{II}$ compositions with $\mathrm{As} / \mathrm{S}$ greater than 1. Thus, the compositional GCss II data plotted in $\mathrm{NiAsS}-\mathrm{CoAsS}-\mathrm{Fe} A s \mathrm{~S}$ space are located within the $600^{\circ} \mathrm{C}$ immiscibility region (Fig. 10B).

During stage $\mathrm{V}$, the activity of arsenic in the mineralizing fluids was as high as in stage IV, with the for- mation of skutterudite II. Both mineralogy and petrography from stage VI support a low-temperature deposition. The assemblage bismuthinite - native bismuth pyrite indicates temperatures close to $271^{\circ} \mathrm{C}$ (melting point of native bismuth), with $\log f\left(\mathrm{~S}_{2}\right)$ of $c a$. -10 .

In spite of a temperature of formation of approximately $600^{\circ} \mathrm{C}$ for the San Juan de Plan deposit deduced from phase relations (Yund 1962, Klemm 1965b, Hem \& Makovicky 2004), the field data do not support such a high temperature. Subsequent geochemical studies on black shales, along with infrared microthermometry studies currently underway, should be able to test whether the deduced evolution of temperature in this study represent "true" conditions of deposition or a later process of re-equilibrium at low temperatures.

Jochum (2000) showed that the precipitation of sulfides minerals in vein systems is probably related to thermochemical processes of sulfate reduction during interaction of the hydrothermal fluids with organic matter in the wallrock. The distinctive spatial association of the $\mathrm{Co}-\mathrm{Ni}-\mathrm{Fe}$ enrichments in the San Juan de Plan ore with the Silurian black shales suggests a genetic link between the style of mineralization and this particular host-rock. The abundant organic matter in the black shales could have acted as a reducing agent for dissolved oxidized sulfur and arsenic species in the hydrothermal fluids, which would have resulted in the deposition of $\mathrm{Co}-\mathrm{Ni}-\mathrm{Fe}$ triarsenides, diarsenides, arsenides and sulfarsenides in close proximity to the contact between the ankerite-rich horizon and the top of Silurian black shales.

\section{Conclusions}

The present study reveals the existence of some discrepancies between our results and those reported in the literature for the system $\mathrm{Co}-\mathrm{Ni}-\mathrm{Fe}-\mathrm{As}-\mathrm{S}$. These discrepancies pertain to the following themes. 
a) The limited miscibility between gersdorffite and rammelsbergite. Electron-microprobe analyses seem to show that there exists a compositional continuum between rammelsbergite and gersdorffite. However, according to our microscope observations, the presence of rammelsbergite in San Juan de Plan ores may be due to the inversion of krutovite after rammelsbergite. Consequently, our findings support those of Spiridonov \& Chvileva (1995) on the complete solid-solution between gersdorffite and krutovite.

b) An almost fixed $\left(\mathrm{Fe}_{0.33} \mathrm{Ni}_{0.67}\right) \leftrightarrow$ Co trend for solid solutions of cobaltite-gersdorffite. Gersdorffitecobaltite $_{\text {ss }}$ from the San Juan de Plan deposit shows the largest variations in Ni:Fe ratio found in the literature, which indicates that $\mathrm{Fe}$ and $\mathrm{Ni}$ are independent of each other. Therefore, the constant $\mathrm{Ni}$ :Fe ratio is not a necessary feature of the ternary sulfarsenides studied.

c) The degree of substitution of As for $\mathrm{S}$ increases with increasing $(\mathrm{Ni}+\mathrm{Fe})$ content. The correlation between $\mathrm{As}_{\text {total }}-\mathrm{As}_{M e A s S}$ and $M e$ reveals the scheme of substitution and, therefore, the compositional trends related to such substitutions. The coupled substitution $(\mathrm{Co}, \mathrm{Fe}) \mathrm{AsS} \leftrightarrow \mathrm{NiAs}_{2}$ in $\mathrm{GC}_{\mathrm{ss}}$ II crystals represents $c a$. $25 \%$ of the total $[$ i.e., $(\mathrm{Co}, \mathrm{Fe}) \mathrm{AsS} \leftrightarrow \mathrm{NiAsS}$ represents ca. 75\%]. However, in $\mathrm{GC}_{\mathrm{ss}}$ I crystals, the substitution CoAsS $\leftrightarrow \mathrm{FeS}_{2}$ is $\mathrm{ca}$. $10 \%$, whereas the substitution $\mathrm{CoAsS} \leftrightarrow \mathrm{CoAs}_{2}$ is $c a .7 \%$. In short, in $\mathrm{GC}_{\mathrm{ss}} \mathrm{I}$ crystals, there is a positive correlation between $\mathrm{S}$ and $\mathrm{Fe}$ contents, and Co and As contents, whereas $\mathrm{GC}_{\mathrm{sS}}$ II crystals show a positive correlation between As and $\mathrm{Ni}$ contents.

d) Natural samples of skutterudite can have a small but real deficiency in As. A representative number of skutterudite samples analyzed from San Juan de Plan do not show such a deficiency.

The formation of the San Juan de Plan deposit can be divided into six stages, as summarized here. Stage I: pyrite I and arsenopyrite precipitate at temperatures between $325^{\circ}$ and $425^{\circ} \mathrm{C}$, with $\log f\left(\mathrm{~S}_{2}\right)$ ranging between -9.2 and -6.2 . Stage II: nickeline, skutterudite I and safflorite were formed, which corresponded to a drop in $f\left(\mathrm{~S}_{2}\right)$. The maximum sulfur content of safflorite from San Juan de Plan may indicate that it formed at temperatures close to $500^{\circ} \mathrm{C}$. Stage III: the crystallization of alloclasite and $\mathrm{GC}_{\mathrm{ss}}$ I crystals was characterized by a metal-rich environment and a high activity of anions, with temperatures close to $600^{\circ} \mathrm{C}$. Stage $I V$ : $\mathrm{GC}_{\mathrm{ss}}$ II crystals, As-Gdf, and rammelsbergite are formed at constant temperatures, whereas the $\mathrm{Ni}$ and anion activities increased. Stage $V$ represents an increase in the activity of As with the precipitation of skutterudite II. Stage VI began with the formation of cobaltite and bismuthinite, which led to the subsequent precipitation of pyrite II, chalcopyrite, and native bismuth at temperatures below $271^{\circ} \mathrm{C}$, and the $\log f\left(\mathrm{~S}_{2}\right)$ was $c a .-10$.

The source of the mineralizing fluid is enigmatic; it could be a residual magmatic fluid migrating through the pile of sedimentary rocks, but further conclusions on the exact nature and genesis of this fluid should be deferred. The abundant organic matter in the black shales of the wallrock could have acted as a reducing agent for dissolved oxidized sulfur and arsenic species in the ore-bearing fluids, which would have resulted in the deposition of $\mathrm{Co}-\mathrm{Ni}-\mathrm{Fe}$ triarsenides, diarsenides, arsenides and sulfarsenides in close proximity to the contact between the ankerite-rich horizon and the top of Silurian black shales.

\section{ACKNOWLEDGEMENTS}

The authors are indebted to E. Makovicky for critical comments and corrections that greatly improved the manuscript. The reviewers, R.A. Ixer, S.R. Hem, R.F. Martin and P.G. Spry, are especially acknowledged for their constructive comments, which greatly contributed to the improvement of the original manuscript. This project was financially supported by Gobierno de Aragon (P2000/013). We thank the Asociación Mineralógica Aragonesa for access to material of private collections. Preliminary studies from San Juan de Plan deposit were undertaken by I.F. and I.S. as a part of a research project at Universidad de Zaragoza (221139).

\section{REFERENCES}

Barkov, A.Y., Thibault, Y., Laajoki, K.V.O., MelezhiK, V.A. \& NiLsson, L.P. (1999): Zoning and substitutions in $\mathrm{Co}-\mathrm{Ni}-(\mathrm{Fe})-\mathrm{PGE}$ sulfarsenides from the Mount General' skaya layered intrusion, Arctic Russia. Can. Mineral. 37, 127-142.

BAYLISS, P. (1982): A further crystal structure refinement of gersdorffite. Am. Mineral. 67, 1058-1064.

BéZiat, D., Monchoux, P. \& Tollon, F. (1996): Cobaltitegersdorffite solid solution as a primary magmatic phase in spessartite, Lacaune area, Montagne Noire, France. Can. Mineral. 34, 503-512.

CAstroviejo, R. \& Nodal, T. (1985): Estudio geológico de las concentraciones de $\mathrm{Co}-(\mathrm{Ni}-\mathrm{Cu}-\mathrm{Fe})$ en el Silúrico de San Juan de Plan, valle de Gistaín (Pirineo Huesca). Bol. Geol. Min. 46, 607-625.

Choi, Seon-Gyn \& ImaI, N. (1985): Ni-Fe-Co arsenides and sulfarsenides from the Ulsan mine, Republic of Korea. Mining Geol. 35, 1-16.

FukUOKa, M. \& HiRowatari, F. (1980): On minerals in the system Ni-Co-As-S from the bedded manganese ore deposits in the eastern part of Yamaguchi Prefecture; on the chemical compositions of gersdorffite-cobaltite solid solution. Sci. Rep. Fac. Sci., Kyushu Univ., Geology 13, 239249.

Gervilla, F., Leblanc, M., Torres-Ruiz, J. \& Fenoll HachALÍ, P. (1996): Immiscibility between arsenide and sulfide melts: a mechanism for concentration of noble metals. Can. Mineral. 34, 485-502. 
Papunen, H., Kojonen, K. \& Johanson, B. (1998) Platinum-palladium- and gold-rich arsenide ores from the Kylmäkoski Ni-Cu deposits (Vammala Nickel Belt, SW Finland). Mineral. Petrol. 64, 163-185.

\& Rønsbo, J. (1992): New data on (Ni,Fe,Co) diarsenides and sulfarsenides in chromite-niccolite ores from Malaga Province, Spain. Neues Jahrb. Mineral., Monatsh., 193-206.

Hem, S.R. (1998): The Ore Mineralogy of the Arroyo de la Cueva Deposits, Ronda Massif, Southeast Spain. Cand. Scient. thesis, Univ. of Copenhagen, Copenhagen, Denmark.

\& MaKovicky, E. (2004): The system Fe-Co-NiAs-S. II. Phase relations in the $(\mathrm{Fe}, \mathrm{Co}, \mathrm{Ni}) \mathrm{As}_{15} \mathrm{~S}_{05}$ section at $650^{\circ}$ and $500^{\circ} \mathrm{C}$. Can. Mineral. 42, 63-86.

\& Gervilla, F. (2001): Compositional trends in $\mathrm{Fe}, \mathrm{Co}$ and $\mathrm{Ni}$ sulfarsenides and their crystalchemical implications; results from the Arroyo de la Cueva deposits, Ronda Peridotite, southern Spain. Can. Mineral. 39, 831-853.

IXeR, R.A., Stanley, C.J. \& Vaughan, D.J. (1979): Cobalt-, nickel-, and iron-bearing sulfarsenides from the north of England. Mineral. Mag. 43, 389-395.

JochUM, J. (2000): Variscan and post-Variscan lead-zinc mineralization, Rhenish Massif, Germany: evidence for sulphide precipitation via thermochemical sulphate reduction. Mineral. Deposita 35, 451-464.

KLEMM, D.D. (1965a): Untersuchung mit der Elektronenstrahlmikrosonde über die natürlichen Mischkristallbereiche der Skutterudite. Contrib. Mineral. Petrol. 11, 322-333.

(1965b): Synthesen und Analysen in den Dreiecksdiagrammen FeAsS-CoAsS-NiAsS und $\mathrm{FeS}_{2}-\mathrm{CoS}_{2}-\mathrm{NiS}_{2}$. Neues Jahrb. Mineral., Abh. 103, 205-255.

KRETSCHMAR, U. \& SCOTT, S.D. (1976): Phase relations involving arsenopyrite in the system $\mathrm{Fe}-\mathrm{As}-\mathrm{S}$ and their application. Can. Mineral. 14, 364-386.

Maurel, C. \& Picot, P. (1974): Stabilité de l'alloclasite et de la cobaltite dans les systèmes $\mathrm{Co}-\mathrm{As}-\mathrm{S}$ et $\mathrm{Co}-\mathrm{Ni}-\mathrm{As}-\mathrm{S}$. Bull. Soc. Fr. Minéral. Cristallogr. 97, 251-256.

MisRa, K. \& FleEt, M.E. (1975): Textural and compositional variations in a Ni-Co-As assemblage. Can. Mineral. 13, $8-14$.

Mroskos, E. (1983): A mineralogical study of the Au-Ag-Bi$\mathrm{Te}-\mathrm{Cu}-\mathrm{Co}-\mathrm{Ni}-\mathrm{As}-\mathrm{S}$ ore mineralization in Macedonia, Greece. Chem. Erde 42, 281-296.

NiCKEL, E.H. (1970): The application of ligand-field concepts to an understanding of the structural stabilities and solid- solution limits of sulphides and related minerals. Chem. Geol. 5, 233-241.

Oen, I.S., Burke, E.A.J., Kieft, C. \& Westerhof, A.B. (1971): $\mathrm{Ni}$-arsenides, Ni-rich loellingite and (Fe,Co)-rich gersdorffite in $\mathrm{Cr}-\mathrm{Ni}$-ores from Malaga Province, Spain. Neues Jahrb. Mineral., Abh. 115, 123-139.

DunN, P.J. \& KIEFT, C. (1984): The nickel-arsenide assemblage from Franklin, New Jersey: description and interpretation. Neues Jahrb. Mineral., Abh. 150, 259-272.

Permingeat, F. \& Zehni, A. (1988): La gersdorffite et la cobaltite des filons d'Oumjerane (Anti-Atlas oriental). Notes Serv. Geol. Maroc 44, 249-251.

Petruk, W., Harris, D.C. \& Stewart, J.M. (1971): Characteristics of the arsenides, sulfarsenides, and antimonides. Can. Mineral. 11, 150-186.

RADCLIFFE, D. \& BERRY, L.G. (1968): The safflorite-löllingite solid solution series. Am. Mineral. 53, 1856-1881.

Roseboom, E.H. (1962): Skutterudites (Co, Ni, Fe)As 3-x $_{\text {- com- }}$ position and cell dimensions. Am. Mineral. 47, 310-327.

(1963): Co-Fe-Ni diarsenides: compositions and cell dimensions. Am. Mineral. 48, 271-299.

RosNeR, B. (1970): Untersuchungen mit der Elektronenstrahlmikrosonde an naturlichen Skutteruditen. Contrib. Mineral. Petrol. 28, 135-146.

Sharp, Z.D., EssENE, E.J. \& Kelly, W.C. (1985): A re-examination of the arsenopyrite geothermometer: pressure considerations and applications to natural assemblages. Can. Mineral. 23, 517-534.

Shengelia, D.M., Akhvlediani, R.A. \& Ketskhoveli, D.N (1977): The graphite geothermometer. Dokl. Acad. Sci. USSR, Earth-Sci. Sect. 235, 132-134.

Spiridonov, E.M. \& ChVILEVA, T.N. (1995). On the boundary between gersdorffite NiAsS and krutovite NiAs. Dokl. Acad. Sci. USSR, Earth-Sci. Sect. 341, 119-123.

Vinogradova, R.A., Krutov, G.A. \& RudashevsKiY, N.S (1974): A variety of nickel alloclasite. Dokl. Akad. Nauk 222, 162-164 (in Russ.).

WAGneR, T. \& LoRenz, J. (2002): Mineralogy of complex Co$\mathrm{Ni}-\mathrm{Bi}$ vein mineralization, Bieber deposit, Spessart, Germany. Mineral. Mag. 66, 385-407.

Yund, R.A. (1962): The system Ni-As-S; phase relations and mineralogical significance. Am. J. Sci. 260, 761-782.

Received January 29, 2004, revised manuscript accepted June 21, 2004. 